\title{
The smallest Dirac eigenvalue in a spin-conformal class and cmc immersions
}

\author{
Bernd Ammann
}

Let us fix a conformal class $\left[g_{0}\right]$ and a spin structure $\sigma$ on a compact manifold $M$. For any $g \in\left[g_{0}\right]$, let $\lambda_{1}^{+}(g)$ be the smallest positive eigenvalue of the Dirac operator $D$ on $(M, g, \sigma)$. In a previous article we have shown that

$$
\lambda_{\min }^{+}\left(M, g_{0}, \sigma\right):=\inf _{g \in\left[g_{0}\right]} \lambda_{1}^{+}(g) \operatorname{vol}(M, g)^{1 / n}>0 .
$$

In the present article, we enlarge the conformal class by adding certain singular metrics. We will show that if $\lambda_{\min }^{+}\left(M, g_{0}, \sigma\right)<$ $\lambda_{\text {min }}^{+}\left(S^{n}\right)$, then the infimum is attained on the enlarged conformal class. For proving this, we solve a system of semi-linear partial differential equations involving a nonlinearity with critical exponent:

$$
D \varphi=\lambda|\varphi|^{2 /(n-1)} \varphi .
$$

The solution of this problem has many analogies to the solution of the Yamabe problem. However, our reasoning is more involved than in the Yamabe problem as the eigenvalues of the Dirac operator tend to $+\infty$ and $-\infty$.

Using the spinorial Weierstraß representation, the solution of this equation in dimension 2 shows the existence of many periodic constant mean curvature surfaces.

\section{Introduction}

Let $M$ be a compact $n$-dimensional manifold, $n \geq 2$, with a fixed conformal class $\left[g_{0}\right]$ and a fixed spin structure $\sigma$. Let $g$ be conformal to $g_{0}$, i.e., $g \in\left[g_{0}\right]$. The classical Dirac operator $D_{g}$ on $(M, g, \sigma)$ has discrete real spectrum with finite multiplicities. The eigenvalues tend to $+\infty$ and $-\infty$. The dimension of the kernel of $D_{g}$ is a spin-conformal invariant, i.e., $\operatorname{dim} \operatorname{ker} D_{g}=$ dim ker $D_{g_{0}}$ for all $g \in\left[g_{0}\right]$. We denote the first (=smallest) positive eigenvalue of $D_{g}$ by $\lambda_{1}^{+}(g)$, and the first (=largest) negative eigenvalue by $\lambda_{1}^{-}(g)$.

Finding bounds for this eigenvalue has attracted much interest during the last decades. Among several estimates [14,21, 22, 24-26] in terms of a 
positive scalar curvature bound, let us mention the following estimate due to Friedrich [14]. If the minimum of the scalar curvature of $(M, g)$ is at least $s>0$, then $\left(\lambda_{1}^{ \pm}(g)\right)^{2} \geq \frac{n}{4(n-1)} s$.

An improvement of this inequality that is important in conformal geometry was derived by Hijazi [18] in terms of the first eigenvalue $\lambda_{1}\left(L_{g}\right)$ of the conformal Laplacian $L_{g}=4 \frac{n-1}{n-2}+\mathrm{scal}_{g}$, namely

$$
\left(\lambda_{1}^{ \pm}(g)\right) \geq \frac{n}{4(n-1)} \lambda_{1}\left(L_{g}\right)
$$

if $n \geq 3$. On the other hand, if the Yamabe invariant

$$
Y\left(M,\left[g_{0}\right]\right)=\inf _{g \in\left[g_{0}\right]} \frac{\int_{M} \mathrm{scal}_{g} \mathrm{dvol}_{g}}{\operatorname{vol}(M, g)^{(n-2) / n}} \in(-\infty, n(n-1)]
$$

is non-negative, one can use the conformal transformation formula for the scalar curvature [12, Theorem 1.159], and one obtains that

$$
\lambda_{1}\left(L_{g}\right) \operatorname{vol}(M, g)^{2 / n} \geq Y\left(M,\left[g_{0}\right]\right) .
$$

Together with (1.1), we get

$$
\left|\lambda_{1}^{ \pm}(g)\right| \operatorname{vol}(M, g)^{1 / n} \geq \sqrt{\frac{n}{4(n-1)} Y\left(M,\left[g_{0}\right]\right)} .
$$

That this inequality even holds in dimension $n=2$, was proved by Bär [9]. In this special case, the Gauss-Bonnet theorem tells us that $Y(M)=4 \pi \chi(M)$, hence we obtain

$$
\left|\lambda_{1}^{ \pm}(g)\right| \operatorname{area}(M, g)^{1 / 2} \geq 2 \sqrt{\pi}
$$

if $M$ is diffeomorphic to $S^{2}$, but we do not get a bound for other surfaces. Equality in (1.2) and (1.3) holds for the round spheres.

Hence, if we know that the value of $Y\left(M,\left[g_{0}\right]\right)$ and if $Y\left(M,\left[g_{0}\right]\right)$ is positive, then we have obtained a positive lower bound $\left|\lambda_{1}^{ \pm}(g)\right| \operatorname{vol}(M, g)^{1 / n}$ that is uniform on the conformal class $\left[g_{0}\right]$. From now on, we will restrict to the first positive eigenvalue, in order to simplify the presentation (see the remark below).

For any compact Riemannian spin manifold $\left(M, g_{0}\right)$ with invertible Dirac operator, the existence of a positive lower bound for $\lambda_{1}^{+}(g) \operatorname{vol}(M, g)^{1 / n}$ was derived by Lott [27]. His estimate does not require that $Y\left(M,\left[g_{0}\right]\right)$ is positive, but needs the weaker assumption that the Dirac operator is invertible. 
Unfortunately, his bound is not explicit, and for most spin-conformal manifolds the determination of the value of the associated infimum

$$
\lambda_{\min }^{+}\left(M,\left[g_{0}\right], \sigma\right):=\inf _{g \in\left[g_{0}\right]} \lambda_{1}^{+}(g) \operatorname{vol}(M, g)^{1 / n}
$$

is still a challenging open problem.

A key idea of Lott's article [27] is to derive a lower bound for the first Dirac eigenvalue in terms of the supremum of a conformally invariant functional, a version of this functional will be explained in Section 2 .

In our article [3], we started to study this functional in more detail. In particular, we showed that Lott's result extends to the case that the Dirac operator has non-trivial kernel. We show that

$$
\lambda_{\min }^{+}\left(M,\left[g_{0}\right], \sigma\right)>0
$$

for any compact Riemannian spin manifold $\left(M,\left[g_{0}\right], \sigma\right)$.

Furthermore, it was shown that

$$
\lambda_{\min }^{+}\left(M,\left[g_{0}\right], \sigma\right) \leq \lambda_{\min }^{+}\left(\mathbb{S}^{n}\right),
$$

where $\mathbb{S}^{n}=\left(S^{n}, g_{\text {can }}\right)$ denotes the sphere with its standard Riemannian metric of constant sectional curvature 1. This bound has been proven in [3] unless $\operatorname{ker} D \neq\{0\}$ and $n=2$. The remaining case ker $D \neq\{0\}$ and $n=2$ was given in [7].

In the present article, we discuss whether the associated infimum in (1.4) is attained. For having a well-behaved minimization problem, it is reasonable to replace the conformal class $\left[g_{0}\right]$ in $(1.4)$ by its $L^{\infty}$-completion $\overline{\left[g_{0}\right]}$. Here, by definition a metric $f^{2} g_{0}$ is in $\overline{\left[g_{0}\right]}$ if $f$ is a real-valued $L^{\infty}$ function. The first positive eigenvalue of the Dirac operator extends naturally to this completion (see Section 3). We show that if we have the strict inequality

$$
\lambda_{\min }^{+}\left(M, g_{0}, \sigma\right)<\lambda_{\min }^{+}\left(\mathbb{S}^{n}\right)=\frac{n}{2} \omega_{n}^{1 / n},
$$

then the infimum is attained by a generalized metric $g \in \overline{\left[g_{0}\right]}$. This minimizer has the form $g:=|\varphi|^{4 /(n-1)} g_{0}$ where $\varphi$ is a spinor of regularity $C^{2}$. The set $\varphi^{-1}(0):=\{x \in M \mid \varphi(x)=0\}$ is called the nodal set of $\varphi$ or the set of degeneration of $g$.

Theorem 1.1. Let $M$ be a compact manifold of dimension $n \geq 2$ with a fixed conformal class $\left[g_{0}\right]$ and a spin structure $\sigma$. Assume that (1.5) holds. Let $\alpha:=2 /(n-1)$ if $n \geq 4$, and let $\alpha \in(0,1)$ if $n \in\{2,3\}$. 
(A) Then there is a spinor field $\varphi \in C^{2, \alpha}(\Sigma M) \cap C^{\infty}\left(\Sigma\left(M \backslash \varphi^{-1}(0)\right)\right)$ on $\left(M, g_{0}\right)$ such that

$$
D_{g_{0}} \varphi=\lambda_{\min }^{+}|\varphi|^{2 /(n-1)} \varphi, \quad\|\varphi\|_{2 n /(n-1)}=1
$$

(B) There is a $g \in \overline{\left[g_{0}\right]}$ such that

$$
\lambda_{1}^{+}(g) \operatorname{vol}(M, g)^{1 / n}=\lambda_{\min }^{+}
$$

The metric has the form $g=|\varphi|^{4 /(n-1)} g_{0}$ where $\varphi$ is a spinor as in (A).

(C) If $\operatorname{dim} M=2$, then the metric $g$ is smooth and the nodal set $\varphi^{-1}(0)$ is finite. Furthermore,

$$
\# \varphi^{-1}(0)<\operatorname{genus}(M)
$$

In particular, if $M$ is diffeomorphic to a 2-torus, then the nodal set $\varphi^{-1}(0)$ is empty.

Inequality (1.5) has been proven if $M$ is conformally flat, if $D$ is invertible, and if the mass endomorphism is not identically zero (after a possible change of orientation if $\operatorname{dim} M \equiv 3 \bmod 4)$ [6]. The mass endomorphism is a section of $\operatorname{End}(\Sigma M)$ defined as the zero-order term of the development of the Green function for the Dirac operator at the diagonal with respect to a conformal coordinate map.

Furthermore, (1.5) is known for many Riemann surfaces (i.e., $n=2$ ), e.g., all rectangular tori have a spin structure such that (1.5) holds.

The Euler-Lagrange equation (1.6) of the above minimization problem has a particularly nice interpretation in dimension $n=2$. Locally, Equation (1.6) can be translated into a conformal constant mean curvature immersion into $\mathbb{R}^{3}$. This translation is a spinorial extension of the Weierstrass representation (see Section 9). By pasting together these local surfaces one obtains a "periodic branched conformal cmc immersion."

More exactly, let $(M, g)$ be a compact Riemann surface together with its universal covering $\pi: \widetilde{M} \rightarrow M$. A periodic branched conformal cmc immersion based on $(M, g)$ is by definition a smooth map $F: \widetilde{M} \rightarrow \mathbb{R}^{3}$ together with finitely many points $p_{1}, \ldots, p_{k} \in M$, the so-called branching points, 
such that the following properties hold:

(1) Periodicity: There is a homomorphism $h: \pi_{1}(M) \rightarrow \mathbb{R}^{3}$, the periodicity map, such that for any $\gamma \in \pi_{1}(M)$, and $x \in \widetilde{M}$ one has

$$
F(x \cdot \gamma)=F(x)+h(\gamma)
$$

Here $\cdot$ denotes the action of $\pi_{1}$ on $\widetilde{M}$ via Deck transformation.

(2) Conformality: The restriction of $F$ to $\widetilde{M} \backslash \pi^{-1}\left(\left\{p_{1}, \ldots, p_{k}\right\}\right)$ is a conformal immersion.

(3) Branching points: We have $d F_{q}=0$ for any $q \in \pi^{-1}\left(\left\{p_{1}, \ldots, p_{k}\right\}\right)$. The order of the first non-vanishing term in the Taylor development of $d F$ in $q$ is called the branching index of $F$ at $q$.

(4) $C M C$ : The image $F\left(\widetilde{M} \backslash \pi^{-1}\left(\left\{p_{1}, \ldots, p_{k}\right\}\right)\right.$ is an immersed surface with constant mean curvature.

The following principle yields the existence of many periodic branched conformal cmc immersions. The set of periodic branched conformal cmc immersions, $H \neq 0$, is essentially (see Section 10 for a precise statement) in bijection with the stationary points of the variational problem associated to our minimization problem. In particular, all minimizers of (1.4) give rise to periodic branched conformal cmc immersions. We obtain

Principle for construction of cmc-surfaces. Assume that the Riemann spin surface $(M, g, \sigma)$ carries a metric $g$ such that the first positive eigenvalue of the Dirac operator is smaller than $2 \sqrt{\pi / \operatorname{area}(M, g)}$. Then there is a periodic branched conformal cmc immersion $F$ based on $(M, g)$. The regular homotopy class of $F$ is determined by the spin-structure $\sigma$. The indices of all branching points are even, and the sum of these indices is smaller than 2genus $(M)$. In particular, if $M$ is a torus, there are no branching points.

Some examples of branched conformal cmc immersions that may arise by this principle are given in Section 10 .

The problem that we are discussing in this article has many relations to the Yamabe problem. For a given compact conformal manifold $\left(M,\left[g_{0}\right]\right)$ the Yamabe problem is the problem to find a metric $g$ in $\left[g_{0}\right]$ of constant scalar curvature. The problem has been affirmatively solved by Trudinger, Aubin, Schoen and Yau, see [29] for a good overview. At first, due to the conformally invariant character of our problem and of the Yamabe problem, certain bounded, but non-compact Sobolev embeddings play an important role. In 
the solution of both problems it is useful to break the conformal invariance by perturbing a parameter. For this perturbed parameter the embeddings are compact, and standard methods yield the existence of a minimizer. It then has to be checked whether the perturbed minimizers converge to a minimizer of the unperturbed problem, or whether they concentrate in some points. In the Yamabe problem the minimizers converge if

$$
Y\left(M,\left[g_{0}\right]\right)<Y\left(\mathbb{S}^{n}\right)
$$

holds. In our variational problem, the minimizers converge if (1.5) holds. Secondly, (1.2) implies that any manifold satisfying (1.5) satisfies (1.7) as well. Hence, proving (1.5) for a given spin-conformal manifold $\left(M,\left[g_{0}\right], \sigma\right)$ solves the Yamabe problem on this manifold.

The structure of the article is as follows. In Section 2 we reformulate the problem of minimizing the first Dirac eigenvalue as a variational problem. We will see that it is natural to admit in infimum (1.4) certain singular metrics namely "metrics" conformal to $g_{0}$ whose conformal factor might vanish somewhere. Such metrics - called generalized metrics - are the subject of Section 3. In the following section, we discuss the round sphere. This example is helpful to obtain a deeper understanding for the analytical difficulties. However, it can be skipped if the reader is only interested in the main results of the article. Section 5 is devoted to certain regularity issues that will become important in Section 6 , where the variational problem is finally solved. We then show in Section 7 how this implies the main theorem. The singularities of the minimizers are discussed in Section 8. In Section 9 we recall the spinorial Weierstrass representation. Section 10 uses the spinorial Weierstrass representation to derive the application to constant mean curvature surfaces. Several examples are included. In Appendix A we summarize (without proofs) some analytical tools. This appendix shall also serve as a reference for fixing the notations for Sobolev spaces and Hölder spaces. In Appendix B we proof a proposition about Hölder spaces, as we could not find a proof in the literature.

Remark (The first negative eigenvalue). The infimum is also attained in (1.4) if we replace $\lambda_{1}^{+}$by $\left|\lambda_{1}^{-}\right|$. In the case $n \not \equiv 3 \bmod 4$ this is obvious: there exists an automorphism of the spinor bundle anticommuting with $D$, hence $\lambda_{1}^{-}(g)=-\lambda_{1}^{+}(g)$. In the case $n \equiv 3 \bmod 4$, the proof for $\lambda_{1}^{-}$is up to the obvious sign changes completely identical. In almost all references cited in the introduction, all statements for $\lambda_{1}^{+}$also hold for $\left|\lambda_{1}^{-}\right|$and vice versa. The only exception is [6]. 
Historical comment. Most of the results in this article were first published in the author's habilitation [4]. The first preprint version appeared on the arxiv in 2003, a simplified and stronger version in 2006. After the preprint version was available, several young mathematicians published results extending this article or strongly related to it, e.g., Simon Raulot, Nadine Große and Andreas Herman.

\section{The associated variational principle}

The goal of this section is to reformulate the problem of minimizing the first Dirac eigenvalue in a conformal class as a variational problem. The choice of a good functional is not very easy, as we would like to find a functional which is both bounded and conformally invariant. The Dirac operator $D$ has a simple behavior under conformal change of the metric, its square $D^{2}$ transforms in a more complicated way. Hence, it is desirable to use a functional that contains only terms in $D \varphi$ and $\varphi$ and no term depending on $D^{2} \varphi$. The $\mathcal{F}_{q}$-functional defined in $(2.1), q=2 n /(n+1)$, is such a conformally invariant, bounded functional, and it will turn out that by working with this functional we obtain the desired results.

Remark. In case, that the reader of our article is already familiar with the analytical problems of the Yamabe problem, he might find it enlightening to compare this problem to our problem. Many techniques from the resolution of the Yamabe problem can be carried over to our setting. However, several arguments from the resolution of the Yamabe problem fail. Two main problems arise: on the one hand the spectrum of $D$ is neither bounded from below nor from above, on the other hand, we are working with sections of a vector bundle instead of functions, hence the standard maximum principle is not available. Such arguments will have to be replaced by other approaches.

At first, we recall some basic definitions and facts from spin geometry. For details we refer to textbooks as for example $[16,28,35]$ or to the beautifully written self-contained introduction [19].

Let $M$ be a compact manifold equipped with a Riemannian metric $g_{0}$ and a spin structure $\sigma$. Assume that $g$ is a metric conformal to $g_{0}$. One associates to $(M, g, \sigma)$ a natural complex vector bundle over $M$ called the spinor bundle $\Sigma(M, g, \sigma) \rightarrow M$. Sections of this bundle are called spinor fields or simply spinors. The bundle carries a hermitian metric, a metric connection and a Clifford multiplication. These additional structures are used to define the Dirac operator $D_{g}: \Gamma(\Sigma(M, g, \sigma)) \rightarrow \Gamma(\Sigma(M, g, \sigma))$, which is a 
first-order elliptic differential operator. The Dirac operator $D_{g}$ is essentially self-adjoint, and hence it has a self-adjoint extension. As a consequence of standard elliptic theory, the spectrum is real and discrete, and all multiplicities are finite.

In the following, the spin structure $\sigma$ will be fixed (and often suppressed in the notation), whereas the metric $g$ varies inside the conformal class $\left[g_{0}\right]$. Some objects will be noted with an index $g$, which means that they are defined with respect to $g$ whereas the same object without the index $g$ indicates that it is defined with respect to the fixed background metric $g_{0}$ (with some exceptions that are apparent from the context). For example, dvol $g$ is the volume element associated to $g$ and $\mathrm{dvol}=\mathrm{dvol}_{g_{0}}$ is the one associated to $g_{0}$. We will frequently use several norms, as for example $\|\varphi\|_{L^{p}}$ and $\|\varphi\|_{H_{1}^{q}}$ that are defined with respect to $g_{0}$ unless otherwise stated. We summarize their definition and some important analytical tools in Appendix A.

The spectrum of the Dirac operator $D_{g}$ will be denoted as

$$
\cdots \leq \lambda_{2}^{-}(g) \leq \lambda_{1}^{-}(g)<0=\cdots=0<\lambda_{1}^{+}(g) \leq \lambda_{2}^{+}(g) \leq \cdots,
$$

where each eigenvalue appears with its multiplicity. Note that 0 may be an eigenvalue or not, and thus $D_{g}$ may be invertible or not. Elliptic theory shows

$$
\lim _{k \rightarrow \infty} \lambda_{k}^{+}(g)=-\lim _{k \rightarrow \infty} \lambda_{-k}^{+}(g)=\infty .
$$

The following transformation formula will be of central importance. To our knowledge, the earliest reference is Hitchin [20]. Another reference written up in a more self-contained manner is [19].

Proposition 2.1 (Conformal transformation formula for D). Let $g=f^{2} g_{0}, f: M \rightarrow \mathbb{R}$ smooth and positive. There is an isomorphism of vector bundles $F: \Sigma\left(M, g_{0}, \sigma\right) \rightarrow \Sigma(M, g, \sigma)$ which is a fiberwise isometry such that

$$
D_{g}(F(\varphi))=F\left(f^{-\frac{n+1}{2}} D_{g_{0}} f^{\frac{n-1}{2}} \varphi\right) .
$$

It is convenient to define

$$
\widetilde{F}(\varphi)=F\left(f^{-\frac{n-1}{2}} \varphi\right) .
$$

We will use this isomorphism $\widetilde{F}$ to identify spinors associated to conformal metrics. With this identification the conformal transformation formula 
reads as

$$
D_{g}(\varphi)=f^{-1} D_{g_{0}}(\varphi)
$$

and $|\varphi|_{g}=f^{-\frac{n-1}{2}}|\varphi|_{g_{0}}$. In particular, with this identification the kernels of $D_{g}$ and $D_{g_{0}}$ coincide. It is easy to verify that with this identification, the expression $\int\left\langle D_{g} \varphi, \varphi\right\rangle_{g} \mathrm{dvol}_{g}$ is conformally invariant. The $L^{p}$-norm $\|\varphi\|_{L^{p}(g)}$ $:=\left(\int|\varphi|_{g}^{p} \mathrm{dvol}_{g}\right)^{1 / p}$ is conformally invariant if and only if $p$ has the value $p_{D}:=2 n /(n-1)$. Similarly, $\left\|D_{g} \varphi\right\|_{L^{q}(g)}$ is conformally invariant if and only if $q$ has the value $q_{D}:=2 n /(n+1)$. Note that $q_{D}{ }^{-1}+p_{D}{ }^{-1}=1$.

For any $q \in\left[q_{D}, 2\right]$ and for any $H_{1}^{q}$-spinor $\varphi$ that is not in the kernel of the Dirac operator we define

$$
\mathcal{F}_{q}^{g}(\varphi)=\frac{\int\left\langle D_{g} \varphi, \varphi\right\rangle_{g} \mathrm{dvol}_{g}}{\left\|D_{g} \varphi\right\|_{L^{q}(g)}^{2}}, \quad \mu_{q}^{g}:=\mu_{q}(M, g, \sigma):=\sup \mathcal{F}_{q}^{g}(\psi)
$$

and $\mathcal{F}_{q}:=\mathcal{F}_{q}^{g_{0}}, \mu_{q}:=\mu_{q}^{g_{0}}$. The well-definedness of $\mathcal{F}_{q}^{g}$ and some basic properties are given by the following lemma.

Lemma 2.1. Let $q \in\left[q_{D}, \infty\right)$. Let $\varphi$ be a spinor field of regularity $H_{1}^{q}$, $D \varphi \neq 0$. Then, $\mathcal{F}_{q}(\varphi)$ is well-defined and real. Furthermore, $\mathcal{F}_{q}: H_{1}^{q} \backslash$ ker $D \rightarrow \mathbb{R}$ is Fréchet differentiable with derivation given by

$$
d \mathcal{F}_{q}(\varphi)(\psi)=\frac{2}{\|D \varphi\|_{L^{q}}^{2}} \int\left\langle\varphi-\rho_{q, \varphi}|D \varphi|^{q-2} D \varphi, D \psi\right\rangle
$$

where $\rho_{q, \varphi}=\mathcal{F}_{q}(\varphi)\|D \varphi\|_{L^{q}}^{2-q}$. The supremum $\mu_{q}$ is positive and finite.

From the above considerations it is evident that the functional $\mathcal{F}_{q}$ is conformally invariant if and only if $q=q_{D}$.

Proof. Let $\varphi$ be a spinor field of regularity $H_{1}^{q}, D \varphi \neq 0$. Take $p$ with $p^{-1}+$ $q^{-1}=1, q \geq \frac{2 n}{n+1}$. Because of $\varphi \in H_{1}^{q} \hookrightarrow L^{p}$, we see with Hölder's inequality that $\langle D \varphi, \varphi\rangle$ is integrable. Thus, the numerator of $\mathcal{F}_{q}$ is well-defined, and hence $\mathcal{F}_{q}$ is well-defined. The self-adjointness of $D$ implies that $\mathcal{F}_{q}(\varphi)$ is real. Moreover, because $H_{1}^{q} \hookrightarrow L^{p}$ is bounded we see that $\left|\int_{M}\langle D \varphi, \varphi\rangle\right| \leq$ $\|D \varphi\|_{L^{q}}\|\varphi\|_{L^{p}}$ is bounded from above by a multiple of $\|\varphi\|_{H_{1}^{q}}^{2}$. Using Theorem A.2 we obtain $\|\varphi\|_{H_{1}^{q}} \leq C\left(\|D \varphi\|_{L^{q}}+\|\pi(\varphi)\|_{L^{q}}\right)$ where $\pi$ is the $L^{2}$ orthogonal projection onto the kernel of $D$. We see that $\mathcal{F}_{q}$ is bounded on $H_{1}^{q} \cap(\operatorname{ker} D)^{\perp} \backslash\{0\}$. An arbitrary spinor field $\varphi \in H_{1}^{q}$ is written as the sum of $\varphi_{1} \in \operatorname{ker} D$ and a non-zero $\varphi_{2} \perp \operatorname{ker} D$. As $\mathcal{F}_{q}(\varphi)=\mathcal{F}_{q}\left(\varphi_{2}\right)$, we see that $\mu_{q}$ is finite. 
Because of $\varphi \in H_{1}^{q}$, we have $|D \varphi|^{q-2} D \varphi \in L^{p}$. Hence the right-hand side of (2.2) defines a continuous functional on $H_{1}^{q}$. We denote the functional by $\psi \mapsto \operatorname{RHS}_{\varphi}(\psi)$. Similarly, one sees that

$$
\mathcal{F}_{q}(\varphi+\psi)-\mathcal{F}_{q}(\varphi)-\operatorname{RHS}_{\varphi}(\psi) \leq o\left(\|\psi\|_{H_{1}^{q}}\right),
$$

hence $\mathcal{F}_{q}$ is Fréchet differential with derivative $\psi \mapsto \operatorname{RHS}_{\varphi}(\psi)$.

If $\varphi$ is an eigenspinor to a positive eigenvalue, then $\mu_{q} \geq \mathcal{F}_{q}(\varphi)>0$.

Proposition 2.2 (Properties of $\left.\mu_{q}^{g}\right)$. The function $\mu_{q}^{g}:\left[q_{D}, \infty\right) \rightarrow(0, \infty)$ is continuous from the right, and

$$
\mu_{2}(M, g, \sigma)=\left(\lambda_{1}^{+}(g)\right)^{-1} .
$$

Furthermore, if $\operatorname{vol}(M, g)=1$, then $\mu_{q}^{g}$ is non-increasing in $q$.

The function $q \mapsto \mu_{q}^{g}$ is sketched in Figure 1 .

Proof. We assume $\operatorname{vol}(M, g)=1$, the statements for $\operatorname{vol}(M, g) \neq 1$ then follow by rescaling.

That $\mu_{q}^{g}$ is non-increasing follows easily from the Hölder inequality.

In order to show the continuity from the right, let $q \geq q_{D}$ be given. We take a smooth spinor field $\varphi$ such that $\mathcal{F}_{q}^{g}(\varphi) \geq \mu_{q}^{g}-\varepsilon$. Observe that

$$
\mathcal{F}_{q^{\prime}}^{g}(\varphi)=\frac{\left\|D_{g} \varphi\right\|_{L^{q}(g)}^{2}}{\left\|D_{g} \varphi\right\|_{L^{q^{\prime}}(g)}^{2}} \mathcal{F}_{q}^{g}(\varphi) .
$$

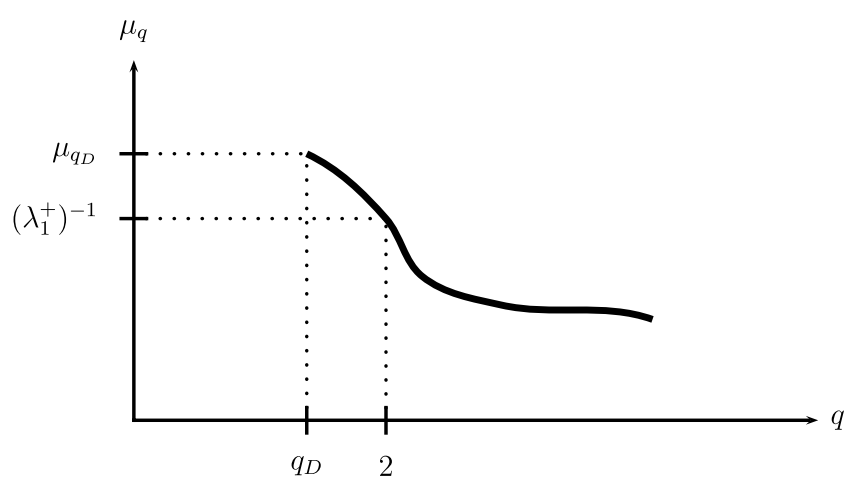

Figure 1: $\mu_{q}$ as a function of $q$. 
The function $q^{\prime} \mapsto\left\|D_{g} \varphi\right\|_{L^{q^{\prime}}(g)}$ is continuous, hence if $q^{\prime} \geq q$ is sufficiently close to $q$, then

$$
\mu_{q^{\prime}}^{g} \geq \mathcal{F}_{q^{\prime}}^{g}(\varphi) \geq \mathcal{F}_{q}^{g}(\varphi)-\varepsilon \geq \mu_{q}^{g}-2 \varepsilon
$$

Because $q \mapsto \mu_{q}^{g}$ is non-increasing, the continuity from the right follows.

The formula $\mu_{2}^{g}=\left(\lambda_{1}^{+}(g)\right)^{-1}$ follows directly if one writes $\varphi$ as a sum of eigenspinors and evaluates $\mathcal{F}_{2}^{g}(\varphi)$.

Remark. In Proposition 6.1 we will see that the supremum defining $\mu_{q}^{g}$ is attained for $q>q_{D}$ by a $C^{2, \alpha}$-spinor and the $C^{1, \alpha}$-norm of the minimizers are uniformly bounded on each compactum in $\left(q_{D}, \infty\right)$. This implies that the function

$$
\left[q_{D}, \infty\right) \rightarrow(0, \infty], \quad q \mapsto \mu_{q}^{g}
$$

is also continuous from the left. We do not carry out this argument in detail as it will not be used later.

\section{Proposition 2.3.}

$$
\mu_{q_{D}}\left(M, g_{0}, \sigma\right)=\frac{1}{\lambda_{\min }^{+}\left(M, g_{0}, \sigma\right)} .
$$

Proof. We have already seen that $\mu_{q_{D}}$ is conformally invariant, i.e., for $g_{1} \in\left[g_{0}\right]$

$$
\mu_{q_{D}}\left(M, g_{0}, \sigma\right)=\mu_{q_{D}}\left(M, g_{1}, \sigma\right)
$$

The previous proposition states that

$$
\mu_{q_{D}}\left(M, g_{1}, \sigma\right) \geq \mu_{2}\left(M, g_{1}, \sigma\right)=\left(\lambda_{1}^{+}\left(g_{1}\right)\right)^{-1}
$$

if $\operatorname{vol}\left(M, g_{1}\right)=1$, and it follows

$$
\mu_{q_{D}}\left(M, g_{0}, \sigma\right) \geq \frac{1}{\lambda_{\min }^{+}\left(M, g_{0}, \sigma\right)} .
$$

It remains to show the inverse inequality which amounts to showing

$$
\sup _{g \in\left[g_{0}\right]} \lambda_{1}^{+}(g)^{-1} \operatorname{vol}(M, g)^{-1 / n} \geq \sup \mathcal{F}_{q_{D}}
$$

For any $\varepsilon>0$ we take a smooth spinor $\varphi_{\varepsilon}$ with $\mathcal{F}_{q_{D}}\left(\varphi_{\varepsilon}\right) \geq \sup \mathcal{F}_{q_{D}}-\varepsilon$ and $\left\|D \varphi_{\varepsilon}\right\|_{L^{q_{D}}}=1$. After a small perturbation of $\varphi_{\varepsilon}$ we can assume that $D \varphi_{\varepsilon}$ 
has no zeros. We set

$$
g_{\varepsilon}:=\left|D \varphi_{\varepsilon}\right|^{4 /(n+1)} g_{0} .
$$

Then we have $\operatorname{vol}\left(M, g_{\varepsilon}\right)=1$, and $\left|D \varphi_{\varepsilon}\right|_{g_{\varepsilon}}$ has constant length 1 . Hence,

$$
\mathcal{F}_{q_{D}}^{\left(M, g_{0}, \sigma\right)}\left(\varphi_{\varepsilon}\right)=\mathcal{F}_{q_{D}}^{\left(M, g_{\varepsilon}, \sigma\right)}\left(\varphi_{\varepsilon}\right)=\mathcal{F}_{2}^{\left(M, g_{\varepsilon}, \sigma\right)}\left(\varphi_{\varepsilon}\right) \leq \mu_{2}\left(M, g_{\varepsilon}, \sigma\right)=\lambda_{1}^{+}\left(g_{\varepsilon}\right)^{-1} .
$$

This implies the proposition.

Now, as we have understood the relationship between the supremum of $\mathcal{F}_{q_{D}}$ and the infimum of $\lambda_{1}^{+} \mathrm{vol}^{1 / n}$, we want to establish a relationship of the maximizers of $\mathcal{F}_{q_{D}}$ and the minimizers of $\lambda_{1}^{+} \mathrm{vol}^{1 / n}$. This will require some knowledge about the Euler-Lagrange equation.

One easily sees that

$$
\mathcal{F}_{q}(s \varphi+\psi)=\mathcal{F}_{q}(\varphi)
$$

for any $\psi \in \operatorname{ker} D$ and $s \in \mathbb{R}^{*}$. Hence maximizers of $\mathcal{F}_{q}$ appear in families and it will be convenient to choose a good representative for each family of maximizers.

Lemma 2.2 (Euler-Lagrange equations of $\mathcal{F}_{q}$.). Let $q \in\left[q_{D}, 2\right]$, and choose $p$ with $q^{-1}+p^{-1}=1$. Suppose that $\mathcal{F}_{q}$ has a maximizing spinor $\varphi_{1} \in$ $H_{1}^{q} \backslash \operatorname{ker} D$. Then there is a maximizing spinor $\varphi \in H_{1}^{q} \backslash \operatorname{ker} D, \varphi \in \mathbb{R}^{*} \varphi_{1}+$ ker $D$, such that

$$
D \varphi=\mu_{q}^{-1}|\varphi|^{p-2} \varphi, \quad \varphi \in H_{1}^{q}, \quad\|\varphi\|_{L^{p}}=1
$$

Proof. We normalize $\varphi_{1}$ such that $\left\|D \varphi_{1}\right\|_{L^{q}}=1$, thus $\rho_{q, \varphi_{1}}=\mu_{q}$. As $\varphi_{1}$ is a maximizer, $d \mathcal{F}_{q}\left(\varphi_{1}\right): H_{1}^{q} \backslash \operatorname{ker} D \rightarrow \mathbb{R}$ is identically zero. Because of (2.2) we see that $\tau:=\varphi_{1}-\mu_{q}\left|D \varphi_{1}\right|^{q-2} D \varphi_{1} \in L^{p}$ is a weak solution of $D \tau=0$, hence it is smooth and in the kernel of $D$. Then, $\varphi_{2}:=\varphi_{1}-\tau=\mu_{q}\left|D \varphi_{1}\right|^{q-2} D \varphi_{1}$ satisfies $D \varphi_{1}=D \varphi_{2}$ and hence

$$
\varphi_{2}=\mu_{q}\left|D \varphi_{2}\right|^{q-2} D \varphi_{2}, \quad \varphi_{2} \in H_{1}^{q}, \quad\left\|D \varphi_{2}\right\|_{L^{q}}=1 .
$$

Taking norms we obtain

$$
\begin{gathered}
\left|\varphi_{2}\right|=\mu_{q}\left|D \varphi_{2}\right|^{q-1}=\mu_{q}\left|D \varphi_{2}\right|^{q / p}, \\
\left|D \varphi_{2}\right|^{q-2}=\left|D \varphi_{2}\right|^{-(q-1)(p-2)}=\left(\mu_{q}^{-1}\left|\varphi_{2}\right|\right)^{-(p-2)} .
\end{gathered}
$$

Hence $\varphi:=\mu_{q}^{-1} \varphi_{2}$ satisfies $(2.3)$. 


\section{Theorem 2.1.}

(a) Let $\psi$ be a maximizing spinor of $\mathcal{F}_{q_{D}}$, and suppose that $\psi$ is smooth and that $D \psi$ vanishes nowhere. Then $g:=|D \psi|^{4 /(n+1)} g_{0}$ is a smooth metric minimizing $\lambda_{1}^{+} \mathrm{vol}^{1 / n}$ in the conformal class $\left[g_{0}\right]$.

(b) Let $g \in\left[g_{0}\right]$ be a (smooth) metric minimizing $\lambda_{1}^{+} \operatorname{vol}^{1 / n}$, and let $\psi$ be an eigenspinor of $D_{g}$ to the eigenvalue $\lambda_{1}^{+}(g)$, then the length of $|\psi|_{g}$ is constant and $\psi$ maximizes $\mathcal{F}_{q_{D}}$.

\section{Proof.}

(a) Let $\psi$ be a maximizing spinor. According to the previous lemma, there is an $s \in \mathbb{R}^{*}$ and a $\tau \in \operatorname{ker} D$ such that $\varphi:=s \psi+\tau$ satisfies (2.3) with $q=q_{D}$ and $p=p_{D}$. In particular, $|\varphi|^{p_{D}-1}=\mu_{q_{D}}|D \varphi|=s \mu_{q_{D}}|D \psi|$ vanishes nowhere. The metric $g_{\varphi}:=|\varphi|^{\frac{4}{n-1}} g_{0}$ satisfies $\operatorname{vol}\left(M, g_{\varphi}\right)=1$ and $|\varphi|_{g_{\varphi}}=1$. Then

$$
\begin{aligned}
\left(\lambda_{1}^{+}\left(g_{\varphi}\right)\right)^{-1} \geq \mathcal{F}_{2}^{g_{\varphi}}(\varphi) & =\mathcal{F}_{q_{D}}^{g_{\varphi}}(\varphi) \\
& =\mathcal{F}_{q_{D}}^{g_{0}}(\varphi) \\
& =\frac{\mu_{q_{D}}^{-1} \int|\varphi|^{p_{D}}}{\mu_{q_{D}}^{-2}\left(\int|\varphi|^{\left(p_{D}-1\right) q_{D}}\right)^{2 / q_{D}}}=\mu_{q_{D}} .
\end{aligned}
$$

As $\mu_{q_{D}}^{-1}=\lambda_{\min }^{+}$we see that $\lambda_{1}^{+}\left(g_{\varphi}\right) \leq \lambda_{\min }^{+}$, hence $g_{\varphi}$ minimizes $\lambda_{1}^{+} \mathrm{vol}^{1 / n}$. By a simple rescaling argument one sees that

$$
g:=|D \psi|^{4 /(n+1)} g_{0}=\left(\frac{1}{s}\right)^{4 /(n+1)}|D \varphi|^{4 /(n+1)} g_{0}=\left(\frac{\lambda_{\min }^{+}}{s}\right)^{4 /(n+1)} g_{\varphi}
$$

minimizes $\lambda_{1}^{+} \operatorname{vol}^{1 / n}$ as well.

(b) By rescaling we can assume that $\operatorname{vol}(M, g)=1$. Unless otherwise indicated all volume measures, norms, scalar products and Dirac operators in this proof are with respect to $g$. In order to show that $|\psi|_{g}$ is constant, we define

$$
f_{t}:=\frac{1+t|\psi|_{g}^{2}}{\left(\int\left(1+t|\psi|_{g}^{2}\right)^{n}\right)^{1 / n}}
$$

One calculates $\left.\frac{d}{d t}\right|_{t=0} f_{t}=|\psi|_{g}^{2}-\int|\psi|_{g}^{2}$. All metrics $f_{t}^{2} g$ have volume 1 . Hence, as the infimum of $\lambda_{1}^{+} \operatorname{vol}^{1 / n}$ is attained in $g$, we have $\lambda_{1}^{+}(g) \leq$ 
$\lambda_{1}^{+}\left(g_{t}\right)$, and hence

$$
\mu_{2}(M, g, \sigma)=\mathcal{F}_{2}^{g}(\psi) \geq \mathcal{F}_{2}^{f_{t}^{2} g}(\psi)=\frac{\int\langle D \psi, \psi\rangle}{\int f_{t}^{-1}|D \psi|^{2}}
$$

For $t=0$ equality is attained. Hence

$$
\int|D \psi|^{2} \leq \int f_{t}^{-1}|D \psi|^{2}
$$

Using $D \psi=\lambda_{1}^{+}(g) \psi$ and deriving with respect to $t$ yields

$$
0=-\left(\lambda_{1}^{+}(g)\right)^{2} \int\left(|\psi|_{g}^{2}-\int|\psi|_{g}^{2}\right)|\psi|_{g}^{2} .
$$

The right-hand side is equal to $-\left(\lambda_{1}^{+}(g)\right)^{2}\left\||\psi|_{g}^{2}-\int|\psi|_{g}^{2}\right\|_{L^{2}}=0$, hence $|\psi|_{g}$ is constant.

This implies that $\mathcal{F}_{q}^{g}(\psi)$ is independent of $q$, thus

$$
\mathcal{F}_{q_{D}}(\psi)=\mathcal{F}_{2}^{g}(\psi)=\left(\lambda_{1}^{+}(g)\right)^{-1}=\left(\lambda_{\text {min }}^{+}\right)^{-1} .
$$

And hence $\mathcal{F}_{q_{D}}$ attains it supremum in $\psi$.

Remark. Later on, we will see that maximizers of $\mathcal{F}_{q_{D}}$ that vanish nowhere are always smooth.

\section{Generalized metrics}

Unfortunately, we cannot exclude that maximizers of $\mathcal{F}_{q}$ vanish somewhere. Maximizers with zeros correspond to metrics with certain singularities, more precisely to metrics whose conformal factor has zeros. These metrics are the main object of this section. We will summarize some facts about the size of the zero set of maximizers in Section 8.

Roughly speaking, generalized metrics are metrics of the form $f^{2} g_{0}$ where $f \in L^{\infty}, f \geq 0$. However, for technical and formal reasons it is better to use the following definition.

Definition. Let $g_{0}$ be a smooth metric on a compact manifold $M$. A generalized metric is a tuple $(f, g)$ where $g \in\left[g_{0}\right]$ and $f \in L^{\infty}, f \geq 0$. If $h>0$ is smooth, we identify $(f h, g)$ with $\left(f, h^{2} g\right)$. Furthermore we identify $g \in\left[g_{0}\right]$ 
with $(1, g)$. Generalized metrics having a representative of the form $\left(f, g_{0}\right)$ are called conformal to $g_{0}$, and those having a representative $(1, g), g \in\left[g_{0}\right]$ are called regular metrics — they correspond to metrics in the ordinary sense. The set of all generalized metrics conformal to $g_{0}$ is called the $L^{\infty}$ completion $\overline{\left[g_{0}\right]}$ of the conformal class $\left[g_{0}\right]$. The volume of $g \in \overline{\left[g_{0}\right]}$ is defined as $\int f^{n} \operatorname{dvol}_{g_{0}}$. For a generalized metric we say that $f^{-1}(0)$ is the set of degeneration.

Remark. The reader should pay some attention to the following technical difficulty: If $f$ vanishes on an open set, then the $L^{\infty}-(2,0)$-tensor $f^{2} g_{0}$ does not determine (the equivalence class) $\left(f, g_{0}\right)$.

However, despite of this remark and slightly abusing the notation, we will write $f^{2} g_{0}$ instead of $\left(f, g_{0}\right)$. Formally $f^{2} g_{0}$ is a generalized metric in the above sense, not an $L^{\infty}-(2,0)$-tensor.

Let us now assume that $M$ carries a fixed spin structure $\sigma$. For any generalized metric $g=f^{2} g_{0}$ we want to define a spinor bundle and a Dirac operator on $(M, g, \sigma)$ in such a way that the results of the previous section carry over to this generalization. In particular, the functional $\mathcal{F}_{q}$ has to be defined and has to be conformally invariant for $q=q_{D}$.

As a vector bundle the spinor bundle $\Sigma(M, g, \sigma)$ is defined to be $\Sigma\left(M, g_{0}, \sigma\right)$, and due to our identification of spinors for different metrics in a fixed conformal class this construction does only depend on the conformal class $\left[g_{0}\right]$ and not on the metric $g_{0}$ itself. For any $x \in M$ and any spinor $\varphi$ in the fiber of $\Sigma(M, g, \sigma)$ over $x$ we define the pointwise norm

$$
|\varphi|_{g}:= \begin{cases}f(x)^{-\frac{n-1}{2}}|\varphi|_{g_{0}} & \text { if } f(x) \neq 0 \\ \infty & \text { if } f(x)=0 \text { and } \varphi(x) \neq 0 \\ 0 & \text { if } f(x)=0 \text { and } \varphi(x)=0\end{cases}
$$

Again, this norm does not change if we replace the background metric $g_{0}$ by a metric conformal to $g_{0}$. For smooth sections $\varphi$ of $\Sigma(M, g, \sigma)$ such that $|\varphi|_{g}<\infty$ almost everywhere we define the $H_{1}^{2}(M, g, \sigma)$-norm as

$$
\left(\int f^{-1}\left|D_{g_{0}} \varphi\right|_{g_{0}}^{2} \mathrm{dvol}_{g_{0}}\right)^{1 / 2}+\left(\int|\varphi|_{g_{0}}^{p_{D}} \operatorname{dvol}_{g_{0}}\right)^{1 / p_{D}}<\infty
$$

where we used the conventions $0^{-1} r=\infty$ for $r>0$ and $0^{-1} 0=0$. The Sobolev space $H_{1}^{2}(M, g, \sigma)$ is the associated completion. 
Lemma 3.1. There is a natural inclusion

$$
H_{1}^{2}(M, g, \sigma) \hookrightarrow H_{1}^{2}\left(M, g_{0}, \sigma\right)
$$

Proof. Cauchy sequences with respect to the norm $H_{1}^{2}(M, g, \sigma)$ are also Cauchy sequences with respect to $H_{1}^{2}\left(M, g_{0}, \sigma\right)$. Thus we obtain a bounded map $H_{1}^{2}(M, g, \sigma) \rightarrow H_{1}^{2}\left(M, g_{0}, \sigma\right)$. In order to prove injectivity of this map one shows that if $\varphi_{i}$ is a Cauchy sequence in $H_{1}^{2}(M, g, \sigma)$ converging to 0 with respect to $H_{1}^{2}\left(M, g_{0}, \sigma\right)$, then it converges to 0 with respect to the $H_{1}^{2}(M, g, \sigma)$ as well.

We define the Dirac operator $D_{g}: H_{1}^{2}(M, g, \sigma) \rightarrow L^{2}(M, g, \sigma), D_{g}(\varphi):=$ $f^{-1} D_{g_{0}}(\varphi)$. The spinor $D_{g}(\varphi)$ is well-defined almost everywhere, as

$$
\left\{x \in M \mid D_{g_{0}}(\varphi)(x) \neq 0 \text { and } f(x)=0\right\}
$$

has measure zero. It is easy to verify that all these definitions only depend on the conformal class of $g_{0}$ and not on $g_{0}$ itself. Furthermore, we can reformulate the definition of the above Sobolev space as

$$
H_{1}^{2}(M, g, \sigma)=\left\{\left.\varphi \in \Gamma(\Sigma(M, g, \sigma))\left|\int\right| D_{g} \varphi\right|_{g} ^{2} \operatorname{dvol}_{g}<\infty\right\} .
$$

We now extend the definitions $\mathcal{F}_{2}^{g}$ and $\lambda_{1}^{+}(g)$ to the $L^{\infty}$-completion of the conformal class.

Definition. For $g=f^{2} g_{0} \in \overline{\left[g_{0}\right]}$ and any $\varphi \in H_{1}^{2}(M, g, \sigma) \backslash \operatorname{ker} D$ we define

$$
\mathcal{F}_{2}^{g}(\varphi):=\frac{\int\left\langle D_{g_{0}} \varphi, \varphi\right\rangle_{g_{0}} \mathrm{dvol}_{g_{0}}}{\int f^{-1}\left|D_{g_{0}} \varphi\right|_{g_{0}}^{2} \mathrm{dvol}_{g_{0}}}
$$

Because of

$$
\begin{aligned}
\int f^{-1}\left|D_{g_{0}} \varphi\right|_{g_{0}}^{2} \operatorname{dvol}_{g_{0}} & \geq \frac{1}{\|f\|_{L^{\infty}}} \int\left|D_{g_{0}} \varphi\right|_{g_{0}}^{2} \operatorname{dvol}_{g_{0}} \\
\mathcal{F}_{2}^{g}(\varphi) & \leq\|f\|_{L^{\infty}} \mathcal{F}_{2}^{g_{0}}(\varphi),
\end{aligned}
$$

the functional is well-defined and bounded on $H_{1}^{2}(M, g, \sigma) \backslash \operatorname{ker} D$.

It is also not hard to see that the supremum is attained. In fact, let $\left(\varphi_{i}\right)$ be a sequence of spinors in $H_{1}^{2}(M, g, \sigma) \backslash \operatorname{ker} D$ with $\mathcal{F}_{2}^{g}\left(\varphi_{i}\right) \rightarrow \sup \mathcal{F}_{2}^{g}$, normalized such that $\int f^{-1}\left|D \varphi_{i}\right|^{2}=1$. Then a subsequence $\left(\varphi_{i_{k}}\right)$ converges weakly in $H_{1}^{2}(M, g, \sigma)$, weakly in $H_{1}^{2}\left(M, g_{0}, \sigma\right)$ and strongly in $L^{2}\left(M, g_{0}, \sigma\right)$ towards a $\varphi_{\infty} \in H_{1}^{2}(M, g, \sigma) \backslash \operatorname{ker} D$. Hence, $\lim \mathcal{F}_{2}^{g}\left(\varphi_{i_{k}}\right) \leq \mathcal{F}_{2}^{g}\left(\varphi_{\infty}\right)$. Thus, 
the supremum is attained in $\varphi_{\infty}$. In analogy to Lemma 2.1 one gets for any smooth test spinor $\psi$

$$
\int\left\langle f^{-1} D \varphi-\left(\mathcal{F}_{2}^{g}(\varphi)\right)^{-1} \varphi, D \psi\right\rangle,
$$

which implies $\tau:=D_{g} \varphi-\left(\mathcal{F}_{2}^{g}(\varphi)\right)^{-1} \varphi \in$ ker $D$ and finally for $\widetilde{\varphi}_{1}:=\varphi_{\infty}+$ $\mathcal{F}_{2}^{g}(\varphi) \tau$

$$
D_{g} \widetilde{\varphi}_{1}=\left(\mathcal{F}_{2}^{g}\left(\widetilde{\varphi}_{1}\right)\right)^{-1} \widetilde{\varphi}_{1}
$$

Definition. The first positive Dirac eigenvalue of $(M, g, \sigma), g=f^{2} g_{0}$ $\in \overline{\left[g_{0}\right]}$ is

$$
\lambda_{1}^{+}(g):=\left(\sup \left\{\mathcal{F}_{2}^{g}(\varphi) \mid \varphi \in H_{1}^{2}(M, g, \sigma) \backslash \operatorname{ker} D\right\}\right)^{-1}
$$

A non-trivial spinor with

$$
D_{g} \varphi=\lambda_{1}^{+}(g) \varphi
$$

is an eigenspinor to the eigenvalue $\lambda_{1}^{+}(g)$.

As we have already seen, this definition coincides with the definition of the first positive Dirac eigenvalue and a corresponding eigenspinor if $g$ is regular.

Most of the statements of the previous section still hold in a modified version for generalized metrics, the proofs are nearly identical. For example one can extend Proposition 2.3 to the following.

\section{Proposition 3.1.}

$$
\inf _{g \in\left[g_{0}\right]} \lambda_{1}^{+}(g) \operatorname{vol}(M, g)^{1 / n}=\inf _{g \in \overline{\left[g_{0}\right]}} \lambda_{1}^{+}(g) \operatorname{vol}(M, g)^{1 / n}=\mu_{q_{D}}(M, g, \sigma)^{-1} .
$$

The equation $\inf _{g \in\left[g_{0}\right]} \lambda_{1}^{+}(g) \operatorname{vol}(M, g)^{1 / n}=\mu_{q_{D}}(M, g, \sigma)^{-1}$ is exactly the statement of Proposition 2.3 and the equation $\inf _{g \in \overline{\left[g_{0}\right]}} \lambda_{1}^{+}(g) \operatorname{vol}(M, g)^{1 / n}=$ $\mu_{q_{D}}(M, g, \sigma)^{-1}$ can be proven with exactly the same proof.

Similarly, we obtain an analogue of Theorem 2.1 .

Theorem 3.1. Let $\left(M, g_{0}, \sigma\right)$ be a compact Riemannian spin manifold.

(a) Let $\psi$ be a maximizing spinor of $\mathcal{F}_{q_{D}}$ (with regularity $H_{1}^{\infty}$ ). Then $g:=$ $|D \psi|^{4 /(n+1)} g_{0}$ is a generalized metric minimizing $\lambda_{1}^{+} \mathrm{vol}^{1 / n}$ in $\overline{\left[g_{0}\right]}$, the $L^{\infty}$-completion of the conformal class $\left[g_{0}\right]$. 
(b) Let $g=f^{2} g_{0} \in \overline{\left[g_{0}\right]}$ be a generalized metric minimizing $\lambda_{1}^{+} \operatorname{vol}^{1 / n}$, and let $\psi$ be an eigenspinor of $D_{g}$ to the eigenvalue $\lambda_{1}^{+}(g)$, then the length of $|\psi|_{g}$ is constant on $M \backslash f^{-1}(0)$ and $\psi$ maximizes $\mathcal{F}_{q_{D}}$.

The proof of this theorem is essentially the same as the proof of Theorem 2.1. Later, we will see that any maximizing spinor has regularity $C^{2, \alpha}$, hence $g=|D \psi|^{4 /(n+1)} g_{0}$ is always a generalized metric.

\section{The case of the sphere}

The sphere $\mathbb{S}^{n}=\left(S^{n}\right.$, can $)$ with the round sphere is an important example. On the one hand, the invariant $\lambda_{\min }^{+}$of the round sphere is contained in many equations and inequalities. On the other hand the round sphere has a large conformal group, and hence studying the minimizers on the sphere helps to understand the analytical difficulties. In particular, we will see why the conclusion in Theorem 6.1 does not hold if $\mu_{q_{D}}=\mu_{q_{D}}^{\mathbb{S}^{n}}$. (Recall $q_{D}=$ $2 n /(n+1)$.

The invariant $\lambda_{\min }^{+}\left(\mathbb{S}^{n}\right)$ is not hard to calculate if one uses the fact that the Yamabe invariant of the round sphere

$$
Y\left(\mathbb{S}^{n}\right):=\inf _{g \in[\operatorname{can}]} \frac{\int \mathrm{scal}_{g} \mathrm{dvol}_{g}}{\operatorname{vol}(M, g)^{(n-2) / n}}
$$

is attained for $g=$ can, and hence $Y\left(\mathbb{S}^{n}\right)=n(n-1) \omega_{n}^{2 / n}$, where $\omega_{n}:=$ $\operatorname{vol}\left(\mathbb{S}^{n}\right)$. The Hijazi inequality $(1.1,1.2)$ tells us, that

$$
\lambda_{\min }^{+}\left(\mathbb{S}^{n}\right)^{2} \geq \frac{n}{4(n-1)} Y\left(\mathbb{S}^{n}\right)=\frac{n^{2}}{4} \omega_{n}^{2 / n} .
$$

Recall that the sphere of constant sectional curvature 1 carries a Killing spinor $\psi$ to the constant $-1 / 2$, i.e., it satisfies

$$
\nabla_{X} \psi=-(1 / 2) X \cdot \psi
$$

Note that this condition implies that the length of $\psi$ is constant. Because of $D \psi=(n / 2) \psi$ we obtain

$$
\lambda_{\min }^{+}\left(\mathbb{S}^{n}\right) \leq \lambda_{1}^{+}\left(\mathbb{S}^{n}\right) \operatorname{vol}\left(\mathbb{S}^{n}\right)^{1 / n} \leq \frac{n}{2} \omega_{n}^{1 / n}
$$

And then

$$
\lambda_{\min }^{+}\left(\mathbb{S}^{n}\right)=\frac{n}{2} \omega_{n}^{1 / n}
$$


The above Killing spinor satisfies

$$
\left(\mathcal{F}_{q_{D}}(\psi)\right)^{-1}=\frac{n}{2} \omega_{n}^{1 / n}\left(\sup \mathcal{F}_{q_{D}}\right)^{-1}
$$

hence $\mathcal{F}_{q_{D}}$ attains its supremum in such Killing spinors.

Let $A: \mathbb{S}^{n} \rightarrow \mathbb{S}^{n}$ be an orientation preserving conformal diffeomorphism. Then the pullback of any spinor $\varphi$ with respect to $A$ is a section of $A^{*}$ $\left(\Sigma\left(S^{n}\right.\right.$, can $\left.)\right)=\Sigma\left(S^{n}, A^{*}\right.$ can $)$, and as before we identify $\Sigma\left(S^{n}, A^{*}\right.$ can $) \cong$ $\Sigma\left(S^{n}\right.$, can $)$ by using the map $\widetilde{F}$ described after Proposition 2.1. If $\varphi$ is a solution of (2.3), then this pullback, denoted by $A^{*} \varphi$, is a solution of this equation as well. Furthermore if $\varphi$ maximizes $\mathcal{F}_{q_{D}}$, then $A^{*} \varphi$ is also a maximizer. As a consequence, all conformal images of Killing spinors to the constant $-1 / 2$ are maximizers of $\mathcal{F}_{q_{D}}$. The following proposition shows that there are no other maximizers on $\mathbb{S}^{n}$.

Proposition 4.1. If $\psi$ is a spinor of regularity $C^{2}$ that attains the supremum of $\mathcal{F}_{q_{D}}$, then there is a Killing spinor $\varphi$ to the Killing constant $-1 / 2$ and an orientation preserving conformal diffeomorphism $A: \mathbb{S}^{n} \rightarrow \mathbb{S}^{n}$ with $A^{*} \varphi=\psi$.

Later on we will see that any maximizer of regularity $H_{1}^{q}$ with $q>q_{D}$ is even $C^{2}$. Hence the statement also holds under this weaker assumption.

Lemma 4.1. Let $(M, g, \sigma)$ be an arbitrary Riemannian spin manifold (not necessarily complete or compact). Assume that there is a spinor $\psi$ of constant length 1 and with $D \psi=\lambda \psi$. Then

$$
\text { scal }=4 \frac{n-1}{n} \lambda^{2}-4|\widetilde{\nabla} \psi|^{2}
$$

where $\widetilde{\nabla}_{X} \psi:=\nabla_{X} \psi+\frac{\lambda}{n} X \cdot \psi$ denotes the Friedrich connection on spinors.

Proof. The Friedrich connection is a metric connection, hence

$$
0=\frac{1}{2} d^{*} d\langle\psi, \psi\rangle=\operatorname{Re}\left\langle\widetilde{\nabla}^{*} \widetilde{\nabla} \psi, \psi\right\rangle-\langle\widetilde{\nabla} \psi, \widetilde{\nabla} \psi\rangle
$$

The twisted version of the Schrödinger-Lichnerowicz formula yields

$$
\left(D-\frac{\lambda}{n}\right)^{2}=\widetilde{\nabla}^{*} \widetilde{\nabla}+\frac{\text { scal }}{4}-\frac{(n-1) \lambda^{2}}{n^{2}} .
$$


Hence

$$
\left(\lambda-\frac{\lambda}{n}\right)^{2}=\left\langle\widetilde{\nabla}^{*} \widetilde{\nabla} \psi, \psi\right\rangle+\frac{\text { scal }}{4}-\frac{(n-1) \lambda^{2}}{n^{2}} .
$$

We obtain

$$
\frac{n-1}{n} \lambda^{2}=\langle\widetilde{\nabla} \psi, \widetilde{\nabla} \psi\rangle+\frac{\text { scal }}{4} .
$$

Proof of Proposition 4.1. We have to show that the supremum is not attained by any other spinor. For proving this, assume that $\psi$ is a maximizer, $\|\psi\|_{L^{q_{D}}}=1$. As the Dirac operator on $\mathbb{S}^{n}$ has kernel $\{0\}$, the spinor satisfies the Euler-Lagrange equation (2.3).

On the open subset $S^{n} \backslash \psi^{-1}(0)$ we define the metric

$$
g_{1}:=|\psi|^{\frac{4}{n-1}} \text { can }
$$

In this metric (2.3) transforms into a solution of

$$
D_{g_{1}} \psi=\lambda_{\min }^{+} \psi, \quad|\psi|_{g_{1}} \equiv 1
$$

On the one hand, one calculates

$$
\operatorname{scal}_{g_{1}}=4 \frac{n-1}{n-2}|\psi|^{-\frac{n+2}{n-1}} \Delta_{\mathrm{can}}|\psi|^{\frac{n-2}{n-1}}+\operatorname{scal}_{\mathrm{can}}|\psi|^{-\frac{4}{n-1}}
$$

and integration yields

$$
\begin{aligned}
\int_{S^{n} \backslash \psi^{-1}(0)} \operatorname{scal}_{g_{1}} \mathrm{dvol}_{g_{1}}= & \int_{M \backslash \psi^{-1}(0)} 4 \frac{n-1}{n-2}|\psi|^{\frac{n-2}{n-1}} \Delta_{\mathrm{can}}|\psi|^{\frac{n-2}{n-1}} \\
& +n(n-1)|\psi|^{2 \frac{n-2}{n-1}} \mathrm{dvol}_{\mathrm{can}} \\
= & \left.\left.\int_{S^{n}} 4 \frac{n-1}{n-2}|d| \psi\right|^{\frac{n-2}{n-1}}\right|_{\mathrm{can}} ^{2}+n(n-1)|\psi|^{2 \frac{n-2}{n-1}} \mathrm{dvol}_{\mathrm{can}}
\end{aligned}
$$

where the last term arises by partial integration. In order to make this step precise one has to exhaust $M \backslash \psi^{-1}(0)$ by smooth manifolds with boundary and partially integrate over these exhausting manifolds. The boundary terms vanish in the limit, as $\psi \rightarrow 0$ on the boundaries and $d|\psi|$ is bounded. 
It is a standard fact from the resolution of the Yamabe problem (see, e.g., [29]) that any $H_{1}^{2}$ function $f$ satisfies

$$
\frac{\int_{S^{n}} 4 \frac{n-1}{n-2}|d f|^{2}+n(n-1) f^{2} \mathrm{dvol}_{\mathrm{can}}}{\left(\int_{S^{n}} f^{\frac{2 n}{n-2}} \mathrm{dvol}_{\mathrm{can}}\right)^{\frac{n-2}{n}}} \geq Y\left(\mathbb{S}^{n}\right)=n(n-1) \omega_{n}^{2 / n} .
$$

Setting $f:=|\psi|^{\frac{n-2}{n-1}}$, we obtain $\int_{S^{n}} f^{\frac{2 n}{n-2}}=1$, and hence the right-hand side of (4.3) is bounded from below by $n(n-1) \omega_{n}^{2 / n}$.

One the other hand, the previous lemma provides

$$
\operatorname{scal}_{g_{1}} \leq 4 \frac{n-1}{n}\left(\lambda_{\min }^{+}\right)^{2}=n(n-1) \omega_{n}^{2 / n}
$$

and as $\operatorname{vol}\left(S^{n} \backslash \psi^{-1}(0), g_{1}\right)=1$, we see that we must have equality in all inequalities involved, in particular in (4.4). An application of the maximum principle [29] yields that $f$ does not vanish, and hence $\psi$ has no zeros. Furthermore, $g_{1}$ is a metric of constant scalar curvature conformal to $\mathbb{S}^{n}$, and such a metric is necessarily of the form $g_{1}:=A^{*}$ can for an orientation preserving conformal diffeomorphism $A: \mathbb{S}^{n} \rightarrow \mathbb{S}^{n}$. With respect to $g_{1}$ we obtain $\widetilde{\nabla} \psi=0$, hence $\psi$ is a Killing spinor on $\left(S^{n}, g_{1}\right)$. This implies that $\varphi:=\left(A^{-1}\right)^{*} \psi$ is a Killing spinor on $\mathbb{S}^{n}$.

Remark. There are solutions to (2.3) that do not maximize the functional. An easy construction of such a solution is as follows. The map $A: \mathbb{S}^{2}=$ $\mathbb{C} \cup\{\infty\} \rightarrow \mathbb{S}^{2}=\mathbb{C} \cup\{\infty\}, z \mapsto z^{k}, k \in \mathbb{N} \backslash\{0\}$ is conformal with branching points 0 and $\infty$. If $\varphi$ is a solution of $D \varphi=c|\varphi|^{2} \varphi$, then the pullback $\psi:=A^{*} \varphi$ is a solution of $D \psi=c|\psi|^{2} \psi$ on $\mathbb{S}^{2} \backslash\{0, \infty\}$. Here $\psi$ is a section of the pull-backed spinor bundle, which is defined using the pull-backed spin structure. The pull-backed spin structure on $\mathbb{S}^{2} \backslash\{0, \infty\}$ coincides with the standard spin structure iff $k$ is odd. If $k$ is odd, one can show that the extension of $\psi$ by setting $\psi(0)=0$ and $\psi(\infty)=0$ is a solution of $D \psi=c|\psi|^{2} \psi$ on $\mathbb{S}^{2}$. However, $\int|\psi|^{4}=k \int|\varphi|^{4}$. This implies $\mathcal{F}(\psi)=k^{-1 / 3} \mathcal{F}(\varphi)$. Hence, if $\varphi$ is a Killing spinor, and $k \geq 3, k$ odd, then $\psi$ is a non-maximizing solution.

\section{Regularity theorems for the Euler-Lagrange equations}

In this section we want to study solutions of the Euler-Lagrange equations. The first subsection "Removal of singularities" will be used in the following section to extend a solution of $\mathbb{R}^{n}=\mathbb{S}^{n} \backslash\{$ South Pole $\}$ to a solution on $\mathbb{S}^{n}$. 
The second subsection proves that solutions of the Euler-Lagrange equations are $C^{2, p-2}$.

\subsection{Removal of singularities}

Theorem 5.1 (Removal of singularities theorem). Let $p \in\left[\frac{n}{n-1}, \infty\right)$. Let $(U, g)$ be a (not necessarily complete) Riemannian manifold equipped with a spin structure, let $x \in U$. Assume that $\varphi \in L^{p}(\Sigma(U \backslash\{x\}), g)$ satisfies weakly on $U \backslash\{x\}$ the equation

$$
D \varphi=\lambda|\varphi|^{p-2} \varphi .
$$

Then this equation even holds weakly on $U$. In particular, the distribution $D \varphi$ does not have singular support in $x$ and is contained in $L^{q}$.

Proof. Let $\psi$ be a smooth spinor compactly supported in $U$. We have to show

$$
\int_{U}\langle\varphi, D \psi\rangle=\lambda \int_{U}\left\langle|\varphi|^{p-2} \varphi, \psi\right\rangle .
$$

For any small $\varepsilon>0$ we choose a smooth cut-off function $\eta_{\varepsilon}: U \rightarrow[0,1]$ with $\eta_{\varepsilon} \equiv 1$ on $B_{\varepsilon}(x)$, with $\left|\nabla \eta_{\varepsilon}\right| \leq 2 / \varepsilon$ and with support in $B_{2 \varepsilon}(x)$. We rewrite the left-hand side as

$$
\begin{aligned}
\int_{U}\langle\varphi, D \psi\rangle & =\int_{U}\left\langle\varphi, D\left(\left(1-\eta_{\varepsilon}\right) \psi+\eta_{\varepsilon} \psi\right)\right\rangle \\
& =\int_{U}\left\langle\varphi, D\left(\left(1-\eta_{\varepsilon}\right)\right) \psi\right\rangle+\int_{U}\left\langle\varphi, \eta_{\varepsilon} D \psi\right\rangle+\int_{U}\left\langle\varphi, \nabla \eta_{\varepsilon} \cdot \psi\right\rangle
\end{aligned}
$$

As $\varphi$ is a weak solution of (5.1) on $U \backslash\{x\}$, the first term equals to

$$
\lambda \int\left\langle|\varphi|^{p-2} \varphi,\left(1-\eta_{\varepsilon}\right) \psi\right\rangle,
$$

and for $\varepsilon \rightarrow 0$ it tends to the right-hand side of (5.2).

Let $q$ be related to $p$ via $1 / q+1 / p=1$. The absolute value of the second term is bounded by

$$
\|\varphi\|_{L^{p}\left(B_{2 \varepsilon}\right)}\|D \psi\|_{L^{q}\left(B_{2 \varepsilon}\right)}
$$

which tends to 0 for $\varepsilon \rightarrow 0$. 
Finally, the absolute value of the third term is bounded by

$$
\frac{2}{\varepsilon}\|\varphi\|_{L^{p}\left(B_{2 \varepsilon}\right)}\|\psi\|_{L^{q}\left(B_{2 \varepsilon}\right)} \leq C\|\varphi\|_{L^{p}\left(B_{2 \varepsilon}\right)} \varepsilon^{\frac{n}{q}-1} .
$$

Our condition $p \geq \frac{n}{n-1}$ yields $q \leq n$, and hence the third term also tends to 0 for $\varepsilon \rightarrow 0$.

\subsection{Regularity}

Theorem 5.2 ( $C^{1, \alpha}$-regularity theorem). Suppose that $\varphi \in H_{1}^{q}, q \in$ $\left[q_{D}, 2\right]$ is a solution of Equations (2.3). Suppose that there is an $r>p_{D}$ such that $\|\varphi\|_{L^{r}}<\infty$. Then $\varphi$ is $C^{1, \alpha}$ for any $\alpha \in(0,1)$.

Furthermore, we obtain a uniform bound of the $C^{1, \alpha}$-norm in the following sense. Let us choose $k, K>0$ such that $\|\varphi\|_{L^{r}}<k$ and $\mu_{q} \geq K$. Then for any $\alpha \in(0,1)$ there is a constant $C$ depending only on $(M, g, \sigma), p, r$, $K, k$ and $\alpha$ with

$$
\|\varphi\|_{C^{1, \alpha}} \leq C
$$

Remark. The following example shows that the theorem cannot hold without the $L^{r}$-bound. Let $M=\mathbb{S}^{n}$ and $p=p_{D}$. Let $\psi$ be a Killing spinor to the Killing constant $-1 / 2$. Suppose $\|\psi\|_{L^{p_{D}}}=1$. Let $A: \mathbb{S}^{n} \rightarrow \mathbb{S}^{n}$ be an orientation preserving conformal diffeomorphism, such that the differential in a given point $x_{0} \in \mathbb{S}^{n}$ satisfies $(d A)_{x_{0}}=2 \mathrm{Id}$. Then $A^{i}$ is again an orientation preserving conformal diffeomorphism with $\left(d A^{i}\right)_{x_{0}}=2^{i}$ Id. It follows that $\psi_{i}=d\left(A^{i}\right) \psi$ is a family of solutions of (2.3), maximizing $\mathcal{F}_{q_{D}}$. However, for any $r>p_{D}$ one can show that $\left\|\psi_{i}\right\|_{L^{r}} \rightarrow \infty$ for $i \rightarrow \infty$. Hence, the $L^{r}$-bound is necessary for the theorem to hold.

Remark. The theorem will be applied in several versions. At first, we will apply it when $p<p_{D}$. In this case the Sobolev embedding $H_{1}^{q} \hookrightarrow L^{r}$, $r=n q /(n-q)>q_{D}$, already provides the required $L^{r}$-bound for $\varphi$. However, the $r$ given by the Sobolev embedding depends on $p$. It will be of central importance to obtain a bound that is uniform for $p \rightarrow p_{D}$. After having proved Theorem 6.1 the uniformity statement in the above theorem will be used to obtain a bound that is uniform for $p \rightarrow p_{D}$. Finally, the regularity theorem will be applied in the case $p=p_{D}$. In this case an additional $L^{r}$-bound is required as well.

Proof of Theorem 5.2. The proof uses the following "bootstrap argument." At first, we assume $r<n \frac{n+1}{n-1}$. As $\varphi$ is $L^{r}$, the right-hand side of (2.3), i.e., 
$|\varphi|^{p-2} \varphi$, is $L^{r /(p-1)} \hookrightarrow L^{s}$ with $s:=r /\left(p_{D}-1\right)=r \frac{n-1}{n+1}<n$. We apply the Global $L^{p}$-estimates A.2 or the Interior $L^{p}$-estimates A.1 with $N:=K:=$ $M$ and get $\varphi \in H_{1}^{s}$. Using the Sobolev embedding I, Theorem A.5(a) one obtains $\varphi \in L^{r^{\prime}}$ with $r^{\prime}=\frac{n s}{n-s}=\frac{r n \frac{n-1}{n+1}}{n-r \frac{n-1}{n+1}}$. Using $r>p_{D}=2 n / n-1$ one sees that $r^{\prime}>r$, hence we have obtained stronger regularity for $\varphi$. We iterate this argument and get $L^{\tilde{r}}$-bounds for arbitrarily large $\tilde{r}$. For any $\tilde{r}>n \frac{n+1}{n-1}$, we obtain $\varphi \in H_{1}^{\tilde{s}}$ with $\tilde{s}:=\tilde{r} /\left(p_{D}-1\right)>n$. We apply the Sobolev embedding theorem II, Theorem A.5(c) and obtain $\varphi \in C^{0, \alpha}$ for any $\alpha \in(0,1)$. Hence $|\varphi|^{p-2} \varphi$ is $C^{0, \alpha}$ as well, and applying Schauder estimates Theorem A.3 we get $\varphi \in C^{1, \alpha}$ for arbitrary $\alpha$.

The uniformity of the upper bound is clear from the construction.

The bootstrap can be continued and we obtain better regularity.

Proposition 5.1 (Improved regularity). We assume the assumptions of the previous theorem.

(1) Let $U:=M \backslash \varphi^{-1}(0)$. Then $\left.\varphi\right|_{U} \in C^{\infty}(U)$.

(2) If $p>2$, then $\varphi \in C^{2, \alpha}$ for any $\alpha \in(0,1) \cap(0, p-2]$. Furthermore,

$$
\|\varphi\|_{C^{2, \alpha}} \leq C
$$

where $C$ depends only on $(M, g, \sigma), p, r, K, k$ and $\alpha$.

(3) If $n=2$ and $p=p_{D}=4$, then $\varphi \in C^{\infty}$. Furthermore,

$$
\|\varphi\|_{C^{m}} \leq C
$$

where $C$ depends only on $(M, g, \sigma), p, r, K, k$ and $m$.

Proof. (1) On $U$ we can continue the bootstrap argument and apply inductively the Schauder estimates Theorem A.3. We conclude that $\varphi$ is smooth on $U$. We obtain (1). Note, that we have not proved that $\|\varphi\|_{C^{m}(U)}$ is finite, as there is no control on high derivatives close to the nodal set.

(2) Let $p>2$. We know that $\varphi$ is $C^{1, \alpha}$ for any $\alpha \in(0,1)$. Hence, using Appendix B one sees that $|\varphi|^{p-2} \varphi$ is $C^{1, \alpha}$ for $\alpha \in(0,1) \cap(0, p-2]$. The Schauder estimates imply that $\varphi$ is $C^{2, \alpha}$.

(3) Similarly, if $n=2$ and $p=4$, then $\varphi \mapsto|\varphi|^{p-2} \varphi$ is also smooth in 0. Hence the bootstrap can go on with higher order Schauder estimates, and we inductively get $\varphi \in C^{m}(M)$ for any $m$. The construction obviously provides uniform bounds. We obtain (3). 


\section{Solution of the variational principle}

Proposition 6.1. Let $q$ and $p$ be related via $1 / p+1 / q=1$. For $q \in\left(q_{D}, 2\right)$ the supremum $\mu_{q}$ of $\mathcal{F}_{q}$ is attained by a spinor field $\varphi \in C^{2, \alpha}, \alpha \in(0,1) \cap$ $(0, p-2], 1 / p+1 / q=1$. The spinor $\varphi$ can be chosen such that $\varphi$ is a solution of $(2.3)$.

Proof. Let $\varphi_{i}$ be a maximizing sequence for $\mathcal{F}_{q}$, i.e., $\mathcal{F}_{q}\left(\varphi_{i}\right) \rightarrow \mu_{q}$. We may assume $\left\|D \varphi_{i}\right\|_{L^{q}}=1$, and that $\varphi_{i}$ is orthogonal to $\operatorname{ker} D$. After taking a subsequence there is a $\varphi_{\infty} \in H_{1}^{q}$ such that $\varphi_{i}$ converges weakly to $\varphi_{\infty}$ in $H_{1}^{q}$. Thus $\left\|D \varphi_{\infty}\right\|_{L^{q}} \leq \liminf \left\|D \varphi_{i}\right\|_{L^{q}}=1$. The compactness of the embedding $H_{1}^{q} \hookrightarrow L^{p}$ provides a subsequence, that converges strongly to $\varphi_{\infty}$ in $L^{p}$. This implies

$$
\int\left\langle D \varphi_{i}, \varphi_{i}\right\rangle=\underbrace{\int\left\langle D \varphi_{i}, \varphi_{i}-\varphi_{\infty}\right\rangle}_{\leq\left\|D \varphi_{i}\right\|_{L^{q}}\left\|\varphi_{i}-\varphi_{\infty}\right\|_{L^{p}}}+\underbrace{\int\left\langle D \varphi_{i}, \varphi_{\infty}\right\rangle}_{\rightarrow \int\left\langle D \varphi_{\infty}, \varphi_{\infty}\right\rangle} \rightarrow \int\left\langle D \varphi_{\infty}, \varphi_{\infty}\right\rangle .
$$

Hence,

$$
\mu_{q}=\lim \mathcal{F}_{q}\left(\varphi_{i}\right)=\lim \frac{\int\left\langle D \varphi_{i}, \varphi_{i}\right\rangle}{\left\|D \varphi_{i}\right\|_{L^{q}}^{2}} \leq \frac{\int\left\langle D \varphi_{\infty}, \varphi_{\infty}\right\rangle}{\left\|D \varphi_{\infty}\right\|_{L^{q}}^{2}}=\mathcal{F}_{q}\left(\varphi_{\infty}\right) \leq \mu_{q} .
$$

As a consequence, we have equality in all inequalities, in particular $\left\|D \varphi_{\infty}\right\|_{L^{q}}$ $=1$. According to Lemma 2.2 one can find $s \in \mathbb{R}^{*}$ and $\tau \in \operatorname{ker} D$ such that $\varphi=s \varphi_{\infty}+\tau$ solves (2.3). Proposition 5.1 (Improved regularity) tells us that $\varphi$ is $C^{2, \alpha}$.

Theorem 6.1 (Uniform $C^{0}$-estimate). Let $\varphi$ be a solution of (2.3) with $p \in\left[2, p_{D}\right)$ and $\mu_{q} \geq \mu_{q_{D}}^{\mathbb{S}^{n}}+\varepsilon, \varepsilon>0$. Then there is a constant $C=C(M, g$, $\sigma, \varepsilon)$ such that

$$
\|\varphi\|_{C^{0}}<C
$$

Remark. The conclusion of this theorem does not hold any longer, if we drop the condition $\mu_{q}>\mu_{q_{D}}^{\mathbb{S}^{n}}$. In fact, if $q=q_{D}$ and $(M, g, \sigma)=\mathbb{S}^{n}$, then there is the following counterexample: Let $A$ be an orientation preserving conformal map $\mathbb{S}^{n} \rightarrow \mathbb{S}^{n}$ with a repelling fixpoint $x_{0}$ as described in the first remark in Subsection 5.2. Let $\varphi$ be a Killing spinor. As seen in the previous section $\varphi$ is a maximizer of $\mathcal{F}_{q_{D}}$ and a solution of (2.3). The images $\varphi_{k}:=\left(A^{k}\right)_{*} \varphi$ under the conformal maps $A^{k}$ are also maximizers of $\mathcal{F}_{q_{D}}$ and 
solutions of (2.3). However, as easily seen, $\left\|\varphi_{k}\right\|_{C^{0}}$ tends to $\infty$ for $k \rightarrow \infty$.

Proof of Theorem 6.1. Assume that such a constant does not exist. Then we find a sequence of solutions $\varphi_{k}$ of

$$
D \varphi_{k}=\mu_{q_{k}}^{-1}\left|\varphi_{k}\right|^{p_{k}-2} \varphi_{k}, \quad \varphi_{k} \in H_{1}^{q_{k}}, \quad\left\|\varphi_{k}\right\|_{L^{p_{k}}}=1
$$

$1 / q_{k}=1-1 / p_{k}, \mu_{q_{k}} \geq \mu_{q_{D}}^{\mathbb{S}^{n}}+\varepsilon$ and

$$
\left\|\varphi_{k}\right\|_{C^{0}} \rightarrow \infty
$$

Let us assume for a moment that $p_{\infty}:=\liminf p_{k}<p_{D}$. In this case, we can choose a subsequence with $p_{k} \rightarrow p_{\infty}$. We have $1=\left\|\varphi_{k}\right\|_{L^{p_{k}}}^{p_{k}}$ $=\left\|\left|\varphi_{k}\right|^{p_{k}-2} \varphi_{k}\right\|_{L^{q_{k}}}^{q_{k}}=\mu_{q_{k}}^{q_{k}}\left\|D \varphi_{k}\right\|_{L^{q_{k}}}^{q_{k}}$. We conclude that $\varphi_{k}$ is bounded in $H_{1}^{\tilde{q}}$ for a $\tilde{q}>q_{D}$, and hence in $L^{r}$ for an $r>p_{D}$. Then the regularity theorem (Theorem 5.2) says that $\left\|\varphi_{k}\right\|_{C^{0}}$ is bounded, in contradiction to (6.2). Hence, the case $p_{\infty}<p_{D}$ cannot occur, i.e., $\lim p_{k}=p_{D}$.

There is a sequence of points $s_{k} \in M$ with

$$
m_{k}:=\left|\varphi_{k}\left(s_{k}\right)\right|=\max \left\{\left|\varphi_{k}(x)\right| \mid x \in M\right\} \rightarrow \infty .
$$

The idea is to blow up suitably the metric such that we obtain in the limit a solution on Euclidean $\mathbb{R}^{n}$.

We define

$$
\begin{aligned}
\tilde{g}_{k} & :=\left(m_{k}\right)^{2\left(p_{k}-2\right)} g, \\
\tilde{\varphi}_{k} & :=\left(m_{k}\right)^{\left(p_{k}-2\right) \frac{n-1}{2}-1} \varphi_{k} .
\end{aligned}
$$

One easily verifies

$$
\left|\tilde{\varphi}_{k}\left(s_{k}\right)\right|_{\tilde{g}_{k}}=1
$$

and obviously (2.3) transforms into

$$
D_{\tilde{g}_{k}} \tilde{\varphi}_{k}=\frac{1}{\mu_{q_{k}}}\left|\tilde{\varphi}_{k}\right|_{\tilde{g}_{k}}^{p_{k}-2} \tilde{\varphi}_{k}
$$


We calculate

$$
\begin{aligned}
\left\|\tilde{\varphi}_{k}\right\|_{L^{p_{k}\left(\tilde{g}_{k}\right)}}^{p_{k}} & =\mu_{q_{k}} \int\left\langle D_{\tilde{g}_{k}} \tilde{\varphi}_{k}, \tilde{\varphi}_{k}\right\rangle_{\tilde{g}_{k}} \operatorname{dvol}_{\tilde{g}_{k}} \\
& =\mu_{q_{k}} \int\left\langle D_{g} \tilde{\varphi}_{k}, \tilde{\varphi}_{k}\right\rangle \operatorname{dvol}_{g} \\
& =\left(m_{k}\right)^{2\left(\left(p_{k}-2\right) \frac{n-1}{2}-1\right)} \underbrace{\left\|\varphi_{k}\right\|_{L^{p_{k}}(g)}^{p_{k}}}_{=1} .
\end{aligned}
$$

We can assume that $m_{k} \geq 1$. As $p_{k} \leq p_{D}$ implies $\left(p_{k}-2\right) \frac{n-1}{2}-1 \leq 0$, we obtain

$$
\left\|\tilde{\varphi}_{k}\right\|_{L^{p_{k}}\left(\tilde{g}_{k}\right)} \leq 1
$$

The injectivity radius of $\left(M, \tilde{g}_{k}\right)$ tends to infinity, i.e., for any $R>0$ there is a $k_{0}=k_{0}(R) \in \mathbb{N}$ such that for all $k \geq k_{0}$ the exponential map $\exp _{s_{k}} \tilde{g}_{k}$ : $T_{s_{k}} M \rightarrow M$ with respect to $\tilde{g}_{k}$ and based in $s_{k}$ is a diffeomorphism on the ball of radius $R$ around 0 . Now, we identify $\left(T_{s_{k}} M, \tilde{g}_{k}\right)$ with $\left(\mathbb{R}^{n}, g_{\text {eucl }}\right)$. Then $\bar{g}_{k}:=\left(\exp _{s_{k}}^{\tilde{g}_{k}}\right)^{*} \tilde{g}_{k}$ is Riemannian metric on $B_{R}(0)$ that coincides with $g_{\text {eucl }}$ in 0 . In the limit $k \rightarrow \infty$ the metrics $\bar{g}_{k}$ converge to $g_{\text {eucl }}$ in the $C^{\infty}$-topology.

As already said in previous sections, the construction of the spinor bundle, its scalar product and its connection depend on the Riemannian metric (and the spin structure). In order to work out the blowup construction one has to define a pullback of the spinors $\widetilde{\varphi}_{k} \in \Gamma\left(\Sigma\left(M, g_{k}, \sigma\right)\right)$ via normal coordinates and then obtain a spinor in $\Gamma\left(\Sigma\left(B_{R}(0), g_{\text {eucl }}\right)\right)$. In the literature two such pullbacks are used, a pullback construction carried out in [7] and inspired by Bourguignon and Gauduchon [13], or via radial parallel transport (see, e.g., the solution of the index problem in [35] using Getzler rescaling). Both pullbacks can be used here. The pullback in [7] has better approximation properties, and is an important tool if one wants to study fine asymptotics of the blowup. However, the pullback via radial parallel transport is technically simpler to introduce, hence it will be used here.

For $R>0$ let $\Sigma_{0}\left(B_{R}(0), g_{\text {eucl }}\right)$ (resp. $\Sigma_{s_{k}}\left(M, \bar{g}_{k}, \sigma\right)$ ) be the fiber of $\Sigma\left(B_{R}(0), g_{\text {eucl }}\right)$ over 0 (resp. $\Sigma\left(M, \bar{g}_{k}, \sigma\right)$ over $\left.s_{k}\right)$. Let us define the radial vector field $X=r \partial r=\sum x^{i} \partial_{x^{i}}$ on $B_{R}(0) \subset \mathbb{R}^{n}$. Its length is the distance from 0 . For sufficiently large $k$, the exponential map of $\left(M, \bar{g}_{k}\right)$ based in $s_{k}$, denoted by $\exp _{s_{k}} \tilde{g}_{k}$ is a diffeomorphism from $B_{R}(0)$ onto its image. One chooses a (complex) linear isometry $\Sigma_{0}\left(B_{R}(0), g_{\text {eucl }}\right) \rightarrow \Sigma_{s_{k}}\left(M, \bar{g}_{k}, \sigma\right)$. This 
map extends uniquely to a fiber preserving map $A: \Sigma\left(B_{R}(0), g_{\text {eucl }}\right) \rightarrow$ $\Sigma\left(M, \bar{g}_{k}, \sigma\right)$, such that

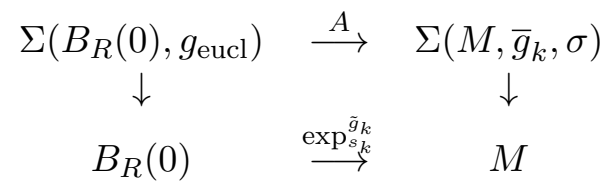

commutes and such that

$$
A\left(\nabla_{X} \varphi\right)=\nabla_{\left(\exp _{s_{k}}^{\tilde{g}_{k}}\right)_{*}(X)} A \varphi .
$$

In a neighborhood of $s_{k}$, this condition can be equivalently characterized by saying that $\varphi \mapsto A \circ \varphi \circ\left(\exp _{s_{k}}^{\tilde{g}_{k}}\right)^{-1}$ maps parallel sections of $\Sigma\left(B_{R}(0), g_{\text {eucl }}\right)$ to radially parallel sections of $\Sigma\left(B_{R}\left(s_{k}\right), \bar{g}_{k}, \sigma\right)$. One easily sees that $A$ is a fiberwise isometry, but the connection on the spinor bundle is not preserved. However, this will not matter, as in the limit $k \rightarrow \infty, R$ fixed, the connections converge in the $C^{\infty}$-topology.

Let $k \geq k_{0}(R)$. On $\left(B_{R}(0), g_{\text {eucl }}\right)$ we define the spinor

$$
\bar{\varphi}_{k}:=A^{-1} \circ \widetilde{\varphi}_{k} \circ \exp _{s_{k}}^{\tilde{g}_{k}}
$$

and the operator $D_{k}: \Gamma\left(\Sigma\left(B_{R}(0), g_{\text {eucl }}\right)\right) \rightarrow \Gamma\left(\Sigma\left(B_{R}(0), g_{\text {eucl }}\right)\right)$,

$$
D_{k}:=A^{-1} \circ D_{\bar{g}_{k}} \circ A,
$$

where $D_{\bar{g}_{k}}$ is the Dirac operator on $\left(B_{R}(0), \bar{g}_{k}\right)$. We obtain

$$
D_{k} \bar{\varphi}_{k}=\frac{1}{\mu_{q_{k}}}\left|\bar{\varphi}_{k}\right|^{p_{k}-2} \bar{\varphi}_{k} .
$$

Note that

$$
\left\|\bar{\varphi}_{k}\right\|_{C^{0}\left(B_{R}(0)\right)} \leq\left|\bar{\varphi}_{k}(0)\right|=1 .
$$

Hence, we may apply the interior $L^{p}$ - and the Schauder-estimates A.1 and A.3 to conclude that

$$
\left\|\bar{\varphi}_{k}\right\|_{C^{1, \alpha}\left(B_{R / 2}(0)\right)} \leq C(R),
$$

with constants $C(R)$ and $k(R)$. The constant $C(R)$ does not depend on $k$ if $\bar{g}_{k}$ is sufficiently $C^{\infty}$-close to $g_{\text {eucl }}$. In particular, the $C^{\infty}$-convergence $\bar{g}_{k} \rightarrow g_{\text {eucl }}$ says that $C(R)$ does not depend on $k$ for $k \geq k_{1}(R)$. 
Compare $D_{k}$ with the Dirac operator $D^{\text {eucl }}$ on Euclidean $\mathbb{R}^{n}$. (For similar and more explicit calculations the reader might consider, e.g., [33] for pullback via radial parallel transport, and [7] for the other pullback method.) We have

$$
\left\|\left(D^{\mathrm{eucl}}-D_{k}\right) \bar{\varphi}_{k}\right\|_{C^{0, \alpha}\left(B_{R / 2}(0), g_{\mathrm{eucl}}\right)} \leq \tau_{k, R}\left\|\bar{\varphi}_{k}\right\|_{C^{1, \alpha}\left(B_{R / 2}(0), \bar{g}_{k}\right)}
$$

with $\lim _{k \rightarrow \infty} \tau_{k, R}=0$. The convergence is not uniform in $R$, but this will not matter in the following.

We choose a sequence of radii $R_{m} \rightarrow \infty$. For each $R_{m}$, the Arcela-Ascoli theorem (Theorem A.4) allows us to choose a subsequence of $\left(\bar{\varphi}_{k}\right)$ converging in $C^{1}\left(B_{R_{m}}(0), g_{\text {eucl }}\right)$. After passing to a diagonal sequence, we see that there is a spinor $\bar{\varphi}_{\infty}$ on $\mathbb{R}^{n}$, such that $\left.\bar{\varphi}_{k}\right|_{B_{R}(0)}$ converges to $\left.\bar{\varphi}_{\infty}\right|_{B_{R}(0)} \in$ $C^{1}\left(B_{R}(0), g_{\text {eucl }}\right)$ for all $R>0$.

Then $\bar{\varphi}_{\infty}$ is a solution of

$$
D^{\mathrm{eucl}} \bar{\varphi}_{\infty}=\frac{1}{\mu_{q_{D}}}\left|\bar{\varphi}_{\infty}\right|^{p_{D}-2} \bar{\varphi}_{\infty}
$$

on $\mathbb{R}^{n}$.

Estimate $(6.5)$ says that $\left\|\widetilde{\varphi}_{k}\right\|_{L^{p_{k}}\left(B_{R}\left(s_{k}\right), \widetilde{g}_{k}\right)} \leq 1$. Hence, for any $\varepsilon>0$ and $R>0$ there is $k_{2}=k_{2}(R, \varepsilon)$ such that

$$
\left\|\bar{\varphi}_{k}\right\|_{L^{p_{k}}\left(B_{R}(0)\right)} \leq 1+\varepsilon
$$

for all $k \geq k_{2}$. Because of the $C^{1}$-convergence $\bar{\varphi}_{k} \rightarrow \bar{\varphi}_{\infty}$, Fatou's lemma yields

$$
\left\|\bar{\varphi}_{\infty}\right\|_{L^{p_{D}\left(B_{R}(0)\right)}} \leq 1
$$

for any $R \in(0, \infty)$, and finally for $R=\infty$.

We identify $\bar{\varphi}_{\infty}$ via stereographic projection with an $L^{p_{D}}$-spinor $\widehat{\varphi}_{\infty}$ on $\mathbb{S}^{n} \backslash$ South pole\} with the identification provided by the application $\widetilde{F}$ directly after Proposition 2.1. We obtain

$$
D^{\mathbb{S}^{n}} \widehat{\varphi}_{\infty}=\frac{1}{\mu_{q_{D}}}\left|\widehat{\varphi}_{\infty}\right|^{p_{D}-2} \widehat{\varphi}_{\infty}
$$


and $\left\|\widehat{\varphi}_{\infty}\right\|_{L^{p_{D}}} \leq 1$. The removal of singularities theorem, i.e., Theorem 5.1, says that (6.6) holds on the whole sphere $\mathbb{S}^{n}$.

$$
\begin{aligned}
& \int_{\mathbb{S}^{n}}\left\langle D \widehat{\varphi}_{\infty}, \widehat{\varphi}_{\infty}\right\rangle=\mu_{q_{D}}^{-1}\left\|\widehat{\varphi}_{\infty}\right\|_{L^{p\left(\mathbb{S}^{n}\right)}}^{p_{D}}, \\
& \left\|D \widehat{\varphi}_{\infty}\right\|_{L^{q_{D}\left(\mathbb{S}^{n}\right)}}=\mu_{q_{D}}^{-1}\left\|\left|\widehat{\varphi}_{\infty}\right|^{p_{D}-1} \widehat{\varphi}_{\infty}\right\|_{L^{q_{D}\left(\mathbb{S}^{n}\right)}}=\mu_{q_{D}}^{-1}\left\|\widehat{\varphi}_{\infty}\right\|_{L^{p_{D}\left(\mathbb{S}^{n}\right)}}^{p_{D}-1}, \\
& \mu_{q_{D}}^{\mathbb{S}^{n}} \geq \mathcal{F}_{q_{D}}^{\mathbb{S}^{n}}\left(\widehat{\varphi}_{\infty}\right)=\mu_{q_{D}}\left\|\widehat{\varphi}_{\infty}\right\|_{L^{p_{D}}\left(\mathbb{S}^{n}\right)}^{2-p_{D}} \geq \mu_{q_{D}}
\end{aligned}
$$

which is apparently a contradiction to our assumption $\mu_{q_{D}} \geq \mu_{q_{D}}^{\mathbb{S}^{n}}+\varepsilon$.

Proposition 6.2. If there is a $p_{0}<p_{D}$ and an $r>p_{D}$ such that for all $t \in\left(p_{0}, p_{D}\right)$ there is a solution $\varphi_{t}$ of Equation (2.3) with $p=t, 1 / q+1 / p=1$ and such that $\left\|\varphi_{t}\right\|_{L^{r}}$ is bounded by a constant $C$ independent from $t$, then there is a sequence $t_{i} \rightarrow p_{D}$ such that $\varphi_{t_{i}}$ converges in the $C^{1}$-topology to a solution of Equation (2.3) with $p=p_{D}$.

Proof. For $p$ sufficiently close to $p_{D}$, we know because of Proposition 2.2 that $\mu_{t}$ is bounded from below by a positive constant. Thus, we can apply the regularity theorem (Theorem 5.2) which tells us that $\left(\varphi_{t}\right)$ is uniformly bounded in $C^{1, \alpha}$. Hence, for a sequence $\left(t_{i}\right)$ with $t_{i}<p_{D}$, converging to $p_{D}$, the spinor fields $\varphi_{t_{i}}$ converge in the $C^{1}$-topology to a $C^{1}$-spinor field $\varphi_{p_{D}}$ which is a solution of Equation (2.3) with $p=p_{D}$.

\section{Proof of the main theorem}

In this section we want to prove the main result of this publication, namely Theorem 1.1.

Proof of Theorem 1.1. Proposition 6.1 tells us that for any $q \in\left(q_{D}, 2\right), q_{D}:=$ $2 n /(n+1)$, the functional $\mathcal{F}_{q}$ is attained by a maximizer denoted $\varphi_{q}$ satisfying

$$
D \varphi_{q}=\mu_{q}^{-1}\left|\varphi_{q}\right|^{p-2} \varphi_{q}, \quad \varphi_{q} \in H_{1}^{q}, \quad\left\|\varphi_{q}\right\|_{L^{p}}=1,
$$

where $p$ and $q$ are related via $p^{-1}+q^{-1}=1$. We have assumed that $\lambda_{\text {min }}^{+}(M, g, \sigma)<\lambda_{\text {min }}^{+}\left(\mathbb{S}^{n}\right)$ which is equivalent to $\mu_{q_{D}}>\mu_{q_{D}}^{\mathbb{S}^{n}}$. As the function $q \mapsto \mu_{q}$ is continuous from the right (Proposition 2.2), we see that there is an $\varepsilon>0$ such that $\mu_{q}>\mu_{q_{D}}^{\mathbb{S}^{n}}+\varepsilon$ for $q$ close to $q_{D}$. For such $q$, Theorem 6.1 implies that $\varphi_{q}$ are uniformly bounded in the $C^{0}$-norm, and then we can 
use Theorem 5.2 to conclude that these $\varphi_{q}$ are even uniformly bounded in $C^{1, \alpha}$. The theorem of Arcela-Ascoli (Theorem A.4) implies that there is a sequence $q_{i} \rightarrow q_{D}$ such that $\varphi_{q_{i}}$ converges in the $C^{1}$-norm to a solution $\varphi$ of

$$
D \varphi=\lambda_{\min }^{+}|\varphi|^{p_{D}-2} \varphi, \quad \varphi \in C^{1}, \quad\|\varphi\|_{L^{p_{D}}}=1 .
$$

Theorem 5.2 and Proposition 5.1 then show that $\varphi$ has the desired regularity, and statement $(\mathrm{A})$ is proven.

We will now show that statement (B) follows from statement (A). If we have a solution as in $(\mathrm{A})$, then we set $g_{1}:=f^{2 /(n-1)} g_{0}$ with $f=\langle\varphi, \varphi\rangle$. Note that $\operatorname{vol}\left(M, g_{1}\right)=\int|\varphi|^{2 n /(n-1)}=1$.

The transformation formula for the Dirac operator under conformal changes (Proposition 2.1) implies that there is a spinor $\varphi_{1}$ on $\left(M, g_{1}, \sigma\right)$ such that

$$
D_{g_{1}} \varphi_{1}=\lambda \varphi_{1}, \quad\left|\varphi_{1}\right|_{g_{1}} \equiv 1 .
$$

Then obviously, $\lambda_{1}^{+}\left(g_{1}\right)=\lambda_{\min }^{+}$and (B) follows.

Statement (C) of Theorem 1.1 will follow from Proposition 8.1, proved in the next section.

\section{The nodal set}

In this section we want to study that the zero set of solutions of the EulerLagrange equation

$$
D \varphi=c|\varphi|^{p-2} \varphi
$$

Note that often the zero set of a function $\varphi$ solving an equation of the above type is denoted as the nodal set of $\varphi$.

The following theorem is due to Bär [10] for smooth $P$.

Theorem 8.1 (Nodal sets for Dirac operators). Let $(U, g)$ be a Riemannian manifold and let $\varphi$ be a solution of

$$
D \varphi=P \cdot \varphi
$$

where $P$ is a smooth function. Then the nodal set of $\varphi$ has Hausdorff dimension at most $n-2$.

Unfortunately, (8.1) has not the desired form as $P=c|\varphi|^{p-2}$ is not smooth for $p \notin \mathbb{Z}$, and Bär's proof does not extend to such a $P$. Nevertheless, we conjecture that the theorem also holds for $P=c|\varphi|^{p-2}, p>2$. 
Conjecture 8.1. The nodal set of any solution of (8.1) has Hausdorff dimension at most $n-2$.

If $n=2$ and $p=p_{D}=4$, then we have better regularity. In this case solutions of Equation (8.1) and the corresponding $P=c|\varphi|^{2}$ are smooth. Hence, using [10, Main Theorem], one sees that in this case the nodal set of a solution is a discrete subset. The following proposition controls its cardinality.

Proposition 8.1. On a compact spin surface $(M, g, \sigma)$ of genus $\gamma$ let $\varphi$ be a solution of equation

$$
D \varphi=\lambda|\varphi|^{2} \varphi, \quad\|\varphi\|_{L^{4}}=1 .
$$

Then the number of zeros of $\varphi$ is at most $\gamma-1+\frac{\lambda^{2}}{4 \pi}$.

In particular, this implies part (C) of Theorem 1.1.

Proof. We set $g_{1}:=|\varphi|^{4} g$. Then outside the zero set we know by Lemma 4.1 that the Gauss curvature of $g_{1}$ is at most $\lambda^{2}$. Furthermore $\operatorname{vol}\left(M, g_{1}\right)=1$. Let $\varphi(p)=0$. The integral of the geodesic curvature with respect to $g_{1}$ over small simply closed loop around $p$ is close to $-2\left(2 j_{p}+1\right) \pi$, where $j_{p}$ is the order of the first non-vanishing term in the Taylor expansion of $\varphi$ in $p$. We remove small open disks around the zeros of $\varphi$ from $M$, and we obtain a surface with boundary $M^{\prime}$. With the Gauss-Bonnet theorem we obtain

$$
2 \pi \chi\left(M^{\prime}\right)=\int_{M^{\prime}} K_{g_{1}}+\int_{\partial M^{\prime}} k_{g_{1}} \leq \lambda^{2}-\sum\left(2 j_{p}+1\right) 2 \pi .
$$

And hence $2 \pi(2-2 \gamma)=2 \pi \chi(M) \leq \lambda^{2}-4 \pi \sum j_{p}$, which implies the proposition.

\section{The spinorial Weierstrass representation}

The aim of this section is to recall the spinorial Weierstrass representation.

Weierstrass published a representation of minimal surfaces in $\mathbb{R}^{3}$ in terms of holomorphic functions [39]. His article deals only with local questions, everything is described in a fixed conformal chart of the surface. From a modern (chart free) point of view, it is clear that these holomorphic sections should be interpreted as a section of the spinor bundle, and "holomorphy" translates into a "harmonicity," i.e., the surface is minimal iff the corresponding spinor $\varphi$ satisfies $D \varphi=0$. 
During the 20th century several attempts were undertaken to globalize the Weierstrass representation and to adapt it to arbitrary surfaces. Unfortunately, most approaches replaced Weierstrass' original approach by a formulation in terms of a holomorphic 1-form and a holomorphic function. The corresponding formulae for non-minimal surfaces were quite involved, and hence not very suitable for applications.

An amazing breakthrough was achieved by work of D. Sullivan, R. Kusner, and N. Schmitt around 1990, and independently by U. Abresch. In early 1989, Dennis Sullivan put together some unpublished notes explaining the spinorial character of the Weierstrass representation for minimal surfaces. In spring 1989, Robert Kusner realized that the spinor formalism is not limited to minimal surfaces, but extends to conformal immersions of arbitrary surfaces, as described below. These techniques were presented in Sullivan's CUNY seminar in 1992, and spread around among the experts rapidly. Kusner's results found their continuation in the $\mathrm{PhD}$ thesis of Schmitt [36]. Schmitt found many interesting applications of the spinorial Weierstrass representation. The results of Kusner and Schmitt led to the publications $[23,36]$.

Independently, Abresch developed a spinorial Weierstrass representation for constant mean curvature surfaces. Unfortunately, the resulting document of Abresch, some handwritten lecture notes from a conference in Luminy, were never published.

We also want to mention an earlier result of Pinkall [34]. In the special class of oriented surfaces, Pinkall's result establishes a bijection between regular homotopy classes of immersions of oriented surfaces $M$ and $\mathbb{Z}_{2}$-valued quadratic forms on $H^{1}\left(M, \mathbb{Z}_{2}\right)$. These quadratic forms are in bijection with the spin structures obtained in the spinorial Weierstrass representation.

More recent literature concerning this representation can be found for example in $[2,11,15]$ and in articles by Pinkall, Taimanov, M.U. Schmidt, Morel, Voss and their collaborators. However, this list is far from being exhaustive.

In our exposition we roughly follow $[15,23]$. The setting for the spinorial Weierstrass representation is as follows. Let $M$ be a compact Riemann surface of genus $\gamma$. The vector bundles $\Lambda^{1,0} T^{*} M$ and $\Lambda^{0,1} T^{*} M$ are defined as the complex linear part and the complex anti-linear part of $T^{*} M \otimes_{\mathbb{R}} \mathbb{C}$. The compositions

$$
\begin{aligned}
& I^{1,0}: T^{*} M \rightarrow T^{*} M \otimes_{\mathbb{R}} \mathbb{C} \rightarrow \Lambda^{1,0} M \\
& I^{0,1}: T^{*} M \rightarrow T^{*} M \otimes_{\mathbb{R}} \mathbb{C} \rightarrow \Lambda^{0,1} M
\end{aligned}
$$


of the complexification and the projection on $\Lambda^{1,0} M$ resp. $\Lambda^{0,1} M$ define vector space homomorphisms $T^{*} M \cong \Lambda^{1,0} M$ and $T^{*} M \cong \Lambda^{0,1} M$. These map $I^{1,0}$ and $I^{0,1}$ preserve the natural connections. However, one should pay attention to the fact, that these maps do not preserve lengths, but $2 \mathrm{Re}$ $g\left(I^{1,0}(\alpha), I^{1,0}(\beta)\right)=2 \operatorname{Re} g\left(I^{0,1}(\alpha), I^{0,1}(\beta)\right)=g(\alpha, \beta)$. The maps $2\left(I^{1,0}\right)^{-1}$ resp. $2\left(I^{0,1}\right)^{-1}$ are denoted as the real part, namely $\operatorname{Re}(\alpha)=2\left(I^{1,0}\right)^{-1}(\alpha)$ for $\alpha \in \Gamma\left(\Lambda^{1,0} M\right)$ and the same notation is used in the $(0,1)$ case. Complex conjugation maps $\Lambda^{0,1} M$ to $\Lambda^{1,0} M$ and vice versa.

As the second Stiefel-Whitney-class of $M$ is the $\bmod 2$ reduction of the Euler-class of $T M \rightarrow M$, one sees that $M$ is spin. However, the space of spin structures on $M$ is not unique: it is an affine space for the group $H^{1}\left(M, \mathbb{Z}_{2}\right)=$ $\left(\mathbb{Z}_{2}\right)^{2 \gamma}$, where $\gamma$ denotes the genus of $M$. Hence, there are $4^{\gamma}$ spin structures on $M$.

If a spin structure is fixed, then the associated vector bundle with respect to the standard representation of $S^{1}$ on $\mathbb{C}$ is a complex line bundle $\Sigma^{+} M$ satisfying $\Sigma^{+} M \otimes \Sigma^{+} M \cong \Lambda^{0,1} M$. If we equip $\Sigma^{+} M$ with the natural hermitian metric, we can choose this map such that the hermitian metric (resp. the connection) on $\Lambda^{0,1} M$ is the tensor product metric (resp. tensor product connection).

We define $\Sigma^{-} M$ to be $\Sigma^{+} M$ with the conjugated complex structure. In particular, there is a natural anti-linear conjugation map $\Sigma^{+} M \rightarrow \Sigma^{-} M$, the hermitian product defines a complex bilinear metric contraction $\Sigma^{-} M \otimes$ $\Sigma^{+} M \rightarrow \mathbb{C}$ and $\Sigma^{-} M \otimes \Sigma^{-} M \cong \Lambda^{1,0} M$. In particular, $\Sigma^{-} M \otimes \Lambda^{0,1} M$ $=\Sigma^{-} M \otimes \Sigma^{+} M \otimes \Sigma^{+} M=\Sigma^{+} M$, and hence the Dolbeault operator is a $\operatorname{map} \bar{\partial}: \Gamma\left(\Sigma^{-} M\right) \rightarrow \Gamma\left(\Sigma^{+} M\right)$, and similarly $\bar{\partial}^{*}=-\partial: \Gamma\left(\Sigma^{+} M\right) \rightarrow \Gamma\left(\Sigma^{-} M\right)$.

We define $c^{1,0}$ and $c^{0,1}$ as the compositions

$$
\begin{aligned}
& T M \stackrel{b}{\rightarrow} T^{*} M \stackrel{I^{1,0}}{\rightarrow} \Lambda^{1,0} M=\Sigma^{-} M \otimes \Sigma^{-} M=\operatorname{Hom}_{\mathbb{C}}\left(\Sigma^{+} M, \Sigma^{-} M\right), \\
& T M \stackrel{b}{\rightarrow} T^{*} M \stackrel{I^{0,1}}{\rightarrow} \Lambda^{0,1} M=\Sigma^{+} M \otimes \Sigma^{+} M=\operatorname{Hom}_{\mathbb{C}}\left(\Sigma^{-} M, \Sigma^{+} M\right) .
\end{aligned}
$$

Composing $c^{1,0}$ with complex conjugation yields $c^{1,0}$ and vice versa. One calculates

$$
c^{1,0}(X) c^{0,1}(Y)+c^{1,0}(Y) c^{0,1}(X)=g(\overline{I(X)}, I(Y))+g(\overline{I(Y)}, I(X))=g(X, Y) .
$$

As a consequence, the map

$$
T M \rightarrow \text { End }\left(\Sigma^{+} M \oplus \Sigma^{-} M\right), \quad X \mapsto \sqrt{2}\left(\begin{array}{cc}
0 & c^{0,1}(X) \\
-c^{1,0}(X) & 0
\end{array}\right)
$$

satisfies the Clifford relations. 
One sees, that the sum $\Sigma M:=\Sigma^{+} M \oplus \Sigma^{-} M$ can be identified with the standard spinor bundle on $M$ in such a way that the above map is the Clifford multiplication, and such that $\Sigma^{+} M$ resp. $\Sigma^{-} M$ are the positive resp. negative half-spinors.

The Dirac operator can be written in this notation as

$$
D=\sqrt{2}\left(\begin{array}{cc}
0 & \bar{\partial} \\
-\partial & 0
\end{array}\right): \Gamma\left(\Sigma^{+} \oplus \Sigma^{-}\right) \rightarrow \Gamma\left(\Sigma^{+} \oplus \Sigma^{-}\right) .
$$

Now let us assume that $\varphi=\left(\varphi_{+}, \varphi_{-}\right)$is a solution of $D \varphi=H|\varphi|^{2} \varphi$, where $H$ is a real-valued function on $M$. This means

$$
\begin{gathered}
-\sqrt{2} \partial \varphi_{+}=H|\varphi|^{2} \varphi_{-}, \\
\sqrt{2} \partial \overline{\varphi_{-}}=H|\varphi|^{2} \overline{\varphi_{+}}
\end{gathered}
$$

We define

$$
\alpha:=\sqrt{2}\left(\begin{array}{c}
\varphi_{+} \otimes \varphi_{+}+\overline{\varphi_{-}} \otimes \overline{\varphi_{-}} \\
i \varphi_{+} \otimes \varphi_{+}-i \overline{\varphi_{-}} \otimes \overline{\varphi_{-}} \\
2 i \varphi_{+} \otimes \overline{\varphi_{-}}
\end{array}\right) \in \Gamma\left(\Lambda^{0,1} \otimes_{\mathbb{R}} \mathbb{R}^{3}\right)
$$

Let $\widetilde{M}$ denote the universal covering of $M$, and $\pi_{1}(M)$ the group of Deck transformations.

As $\partial \alpha=d \alpha$ is imaginary, we can find a function $F: \widetilde{M} \rightarrow \mathbb{R}^{3}$, such that $d F=\operatorname{Re} \alpha$, and there is a homomorphism $V: \pi_{1}(M) \rightarrow \mathbb{R}^{3}$, such that

$$
F(p \cdot \gamma)=F(p)+V(\gamma) \quad \forall p \in \widetilde{M}, \gamma \in \pi_{1}(M)
$$

One calculates that $F$ is a conformal map with possible branching points,

$$
|d F|=|\operatorname{Re} \alpha|=\frac{1}{\sqrt{2}}|\alpha|=|\varphi|^{2}
$$

and that $F(M)$ has mean curvature $H$.

Hence, the map $F$ satisfies properties (1) to (3) from the introduction, i.e., it is a periodic branched conformal immersion $F$ based on $(M, g)$ with mean curvature $H$.

In any zero of the spinor $\varphi$, the map $F$ has a branching point. If $F$ vanishes of order $k$, then $\alpha$ vanishes of order $2 k$. Hence, all branching points of $F$ are necessarily of even order. 
Summarizing the above statements, we obtain for any solution of $D \varphi=$ $H|\varphi|^{2} \varphi$ a periodic branched conformal immersion of $\widetilde{M}$ into $\mathbb{R}^{3}$ which is uniquely determined up to translation. If $\varphi$ solves $D \varphi=H|\varphi|^{2} \varphi$, then $-\varphi$ as well, and the corresponding $F$ is the same. Hence, we obtain a well-defined map

$$
\left\{\begin{array}{l}
\text { solutions of } \\
\mathrm{D} \varphi=H|\varphi|^{2} \varphi \\
\text { on } M
\end{array}\right\} / \pm 1 \rightarrow\left\{\begin{array}{l}
\text { conformal periodic } \\
H \text {-immersions } \\
\widetilde{M} \rightarrow \mathbb{R}^{3} \text { with branching } \\
\text { points of even order }
\end{array}\right\} \begin{aligned}
& \text { trans- } \\
& \text { lations }
\end{aligned}
$$

and one can show that this map is even a bijection.

The inverse of this map is given by restricting a parallel spinor on $\mathbb{R}^{3}$ to $F(M)$ and by performing a conformal change [11].

If $H$ is constant, then there is also another version of the spinorial Weierstrass representation, where the target space is $S^{3}$ instead of $\mathbb{R}^{3}$. We view $S^{3}$ as SU(2) with a bi-invariant metric of constant curvature 1, the multiplication in $\mathrm{SU}(2)$ is denoted with $\bullet$. The periodicity condition (1) has to be replaced by

$\left(1^{\prime}\right)$ Left periodicity: There is a homomorphism $h: \pi(M) \rightarrow \mathrm{SU}(2)$, the periodicity map, such that for any $\gamma \in \pi_{1}(M)$, and $x \in \widetilde{M}$ one has

$$
F(x \cdot \gamma)=h(\gamma) \bullet F(x)
$$

Here $\cdot$ denotes the action of $\pi_{1}$ on $\widetilde{M}$ via Deck transformation.

One obtains a bijection

$$
\left\{\begin{array}{l}
\text { solutions of } \\
\mathrm{D} \varphi=c|\varphi|^{2} \varphi \\
\text { on } M
\end{array}\right\} / \pm 1 \longrightarrow\left\{\begin{array}{l}
\text { conformal left periodic } H \text {-im- } \\
\text { mersions } \widetilde{M} \rightarrow \mathrm{SU}(2) \text { with } \\
\text { branching points of even order }
\end{array}\right\} \begin{aligned}
& \text { Left mul- } \\
& \text { tiplication }
\end{aligned}
$$

where $c=\sqrt{H^{2}+1}$. For details see $[4,32,38]$.

\section{Applications to constant mean curvature surfaces}

If the dimension of $M$ is 2 , then (1.6) reads as

$$
D \varphi=\lambda_{\min }^{+}|\varphi|^{2} \varphi, \quad \varphi \in H_{1}^{4 / 3}, \quad\|\varphi\|_{L^{4}}=1
$$


and according to the regularity theory $\varphi$ is even smooth.

The spinorial Weierstrass representation explained in the previous section tells us that such a solution can be used to construct certain immersions with constant mean curvature.

Combining the previous results we obtain the following application that is a stronger version of the "Principle for construction of cmc-surfaces" mentioned in the introduction.

Proposition 10.1. Assume that the Riemann spin surface $(M, g, \sigma)$ satisfies

$$
\lambda_{\min }^{+}(M,[g], \sigma)<2 \pi
$$

Then there is a periodic branched conformal cmc immersion $F: \widetilde{M} \rightarrow \mathbb{R}^{3}$ based on $(M, g)$. The mean curvature is equal to $\lambda_{\min }^{+}(M,[g], \sigma)$ and the area of a fundamental domain is 1 . The regular homotopy class of $F$ is determined by the spin-structure $\sigma$. The indices of all branching points are even, and the sum of these indices is smaller than 2 genus $(M)$. In particular, if $M$ is a torus, there are no branching points.

The proof is a direct consequence of Theorem 1.1 and the previous section.

There are many examples of stationary points of the functional. However, it is still open whether they are the maximizers or not. Note, that by changing the orientation of a surfaces, a maximizers $\psi$ of $\mathcal{F}_{q_{D}}$ on $M$ turns into a minimizer $\psi^{\prime}$ of $\mathcal{F}_{q_{D}}$ on the surface with reversed orientation $M^{\prime}$, with $\mathcal{F}_{q_{D}}^{M^{\prime}}\left(\psi^{\prime}\right)=-\mathcal{F}_{q_{D}}^{M}(\psi)$. Let us study some examples.

\section{Examples.}

(a) Let $(M, g)$ be a two-dimensional torus. Via a conformal change we can achieve that $g$ is flat, i.e., $M=\mathbb{R}^{2} / \Gamma$, equipped with the Euclidean metric. We assume that the lattice $\Gamma$ is generated by $\left(\begin{array}{l}1 \\ 0\end{array}\right)$ and $\left(\begin{array}{l}x \\ y\end{array}\right)$, with $y>0$. The spinor bundle of a flat manifold is flat as well, hence the holonomy is a map $\Gamma \rightarrow \mathrm{SU}\left(\Sigma_{p} M\right)$. Indeed, the image of this map is contained in $\{ \pm \mathrm{Id}\}$. We obtain a homomorphism $\chi: \Gamma \rightarrow\{ \pm \mathrm{Id}\}$. This homomorphisms characterizes the spin structure $\sigma$ in the sense that two spin structures on $(M, g)$ are isomorphic iff the homomorphisms $\chi$ coincide, and to each such homomorphism there is a spin structure. 
The case $\chi \equiv+$ Id corresponds to the so-called trivial spin structure $\sigma_{\mathrm{tr}}$, the other cases correspond to non-trivial spin structures. ${ }^{1}$

At first, we deal with the case $\sigma=\sigma_{\text {tr }}$. In this case, after a possible rotation and a possible homothety, we can achieve

$$
|x| \leq \frac{1}{2}, \quad y^{2}+x^{2} \geq 1, \quad y>0
$$

On $\left(M, g, \sigma_{\mathrm{tr}}\right)$ the kernel of $D$ has complex dimension 2, and consists of parallel spinors. If one carries out the constructions from the last sections for a parallel spinor, then one obtains an affine conformal map $F$. Such an $F$ is trivially a periodic conformal immersion $F$ based on $(M, g)$ with vanishing mean curvature. However, if $y>\pi$ then one easily sees that $\lambda_{\min }^{+}\left(M, g, \sigma_{\mathrm{tr}}\right)<2 \pi$. The proposition yields the existence of a periodic conformal immersion $F$ based on $(M, g)$ with constant mean curvature $\lambda_{\min }^{+}\left(M, g, \sigma_{\mathrm{tr}}\right)$, and the area of a fundamental domain is 1 . However, the proposition only provides the existence of the solution, but we cannot characterize the maximizer. In our example, a family of solutions can be explicitly written, namely it is the family of parametrized cylinders

$$
\begin{aligned}
F & : \mathbb{R}^{2} \rightarrow \mathbb{R}^{3} \\
& \left(\begin{array}{l}
a \\
b
\end{array}\right) \mapsto P\left(\begin{array}{c}
\frac{\sqrt{y}}{4 \pi} \cos \frac{4 \pi b}{y} \\
\frac{\sqrt{y}}{4 \pi} \sin \frac{4 \pi b}{y} \\
\frac{a}{\sqrt{y}}
\end{array}\right)+X_{0}
\end{aligned}
$$

for any $P \in O(3), X_{0} \in \mathbb{R}^{3}$. We conjecture that these solutions are exactly those that correspond to the maximizers and the minimizers of $\mathcal{F}_{q_{D}}$, when we normalize such that all spinors have $L^{4}$-norm 1 .

In the case $\sigma \neq \sigma_{\text {tr }}$ we can achieve that

$$
\begin{gathered}
\chi\left(\begin{array}{l}
1 \\
0
\end{array}\right)=\mathrm{Id}, \quad \chi\left(\begin{array}{l}
1 \\
0
\end{array}\right)=-\mathrm{Id}, \\
|x| \leq \frac{1}{2}, \quad y^{2}+\left(|x|-\frac{1}{2}\right)^{2} \geq \frac{1}{4}, \quad y>0 .
\end{gathered}
$$

The Dirac operator is always invertible.

\footnotetext{
${ }^{1}$ Note that this notation is a bit misleading, as it is only the trivial spin structure on $M$ that defines a non-trivial element in the bordism class.
} 
One easily sees $\lambda_{\min }^{+}(M, g, \sigma) \leq \frac{\pi}{\sqrt{y}}$. Hence, the proposition yields solutions for $y>\frac{4}{\pi}$. Once again, solutions can be explicitly written, namely the parameterized cylinder

$$
\begin{aligned}
F & : \mathbb{R}^{2} \rightarrow \mathbb{R}^{3} \\
& \left(\begin{array}{l}
a \\
b
\end{array}\right) \mapsto P\left(\begin{array}{c}
\frac{\sqrt{y}}{2 \pi} \cos \frac{2 \pi b}{y} \\
\frac{\sqrt{y}}{2 \pi} \sin \frac{2 \pi b}{y} \\
\frac{a}{\sqrt{y}}
\end{array}\right)+X_{0}
\end{aligned}
$$

for any $P \in O(3), X_{0} \in \mathbb{R}^{3}$. However, in some cases, e.g., if $x=0$ and $4 / \pi<y<1$, these solutions no longer correspond to maximizers and minimizers, but to saddle points of the functional. We conjecture that in the case $x=0, y<1$ the maximizers and minimizers correspond to the unduloid immersions (see figure 2). An unduloid is a surface of revolution of constant mean curvature.

(b) If $M$ has genus 2, then as in the case of the torus, the dimension of the kernel is independent of the metric, however it depends on the spin structure. If $\sigma$ is a spin structure such that $(M, \sigma)$ is spin-cobordant 0 , then the Dirac operator is invertible for any metric. Again, as in the torus case, one can find for any $\varepsilon>0$ a conformal classes $g$ on $M$ with $\lambda_{\min }^{+}(M,[g], \sigma)<\varepsilon[5]$

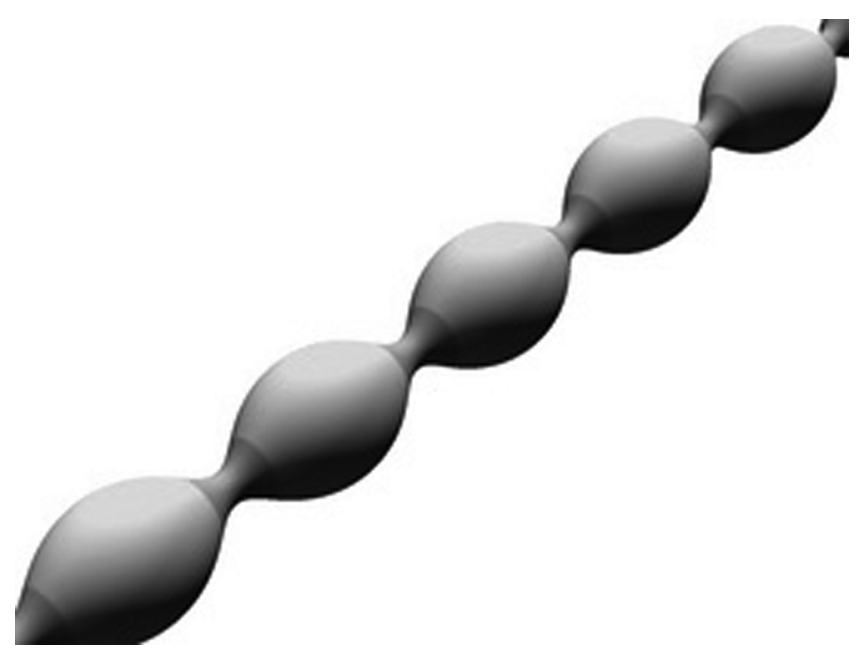

Figure 2: An unduloid in $\mathbb{R}^{3}$, visualized by N. Schmitt, University of Tübingen, Germany. 
(c) If the genus is larger than 2, then the kernel of the Dirac operator on a Riemannian spin manifold $(M, \sigma)$ depends on the metric. For example if $M$ is a surface of genus 3 equipped with the spin structure $\sigma$ and the conformal structure $g_{0}$ associated to the periodic conformal immersion with vanishing mean curvature indicated in figure 3 . This immersion induces a harmonic spinor on $(M, g, \sigma)$. However, as $(M, \sigma)$ is spincobordant 0 , there is a perturbation $\left[g_{t}\right]$ of the conformal structure such that the Dirac operator on $\left(M, g_{t}, \sigma\right)$ has a trivial kernel for small $t \neq 0[31]$. In this case

$$
\lim _{\substack{t \rightarrow 0 \\ t \neq 0}} \lambda_{\min }^{+}\left(M,\left[g_{t}\right], \sigma\right)=0
$$

hence there exist solutions of (10.1). Such a solution is visualized in figure 4 .

(d) Constant mean curvature immersions of $T^{2}$ into $\mathbb{R}^{3}$, in particular Wente tori and twisty tori (figure 5) also correspond to stationary points of $\mathcal{F}_{q_{D}}$. However, as they are not embedded [30] tells us that

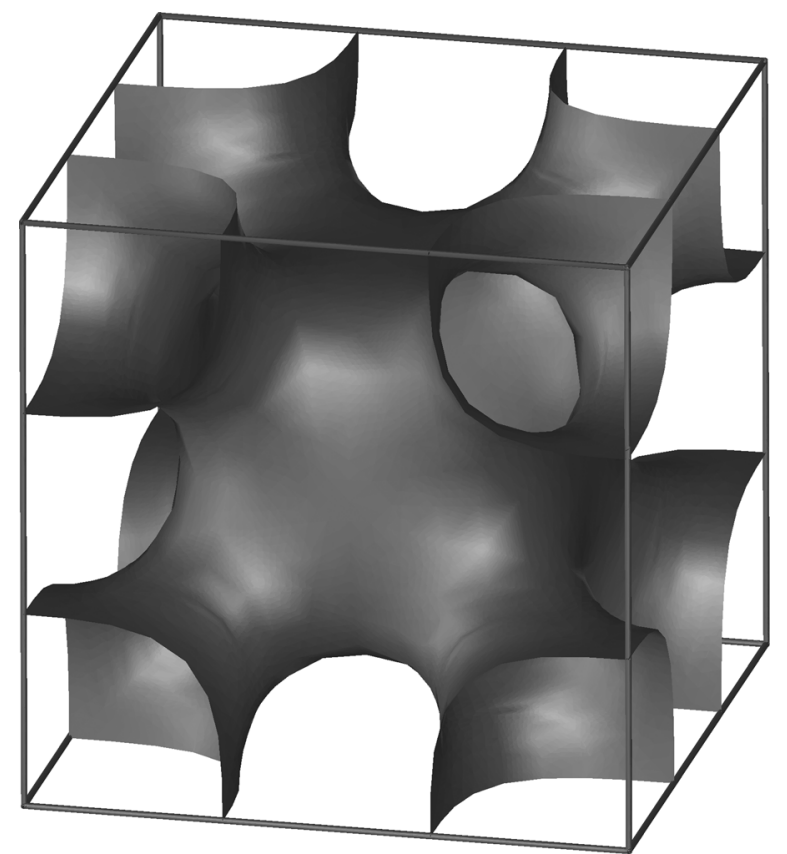

Figure 3: A periodic branched conformal minimal surface, visualized by K. Grosse-Brauckmann, University of Darmstadt, Germany. 


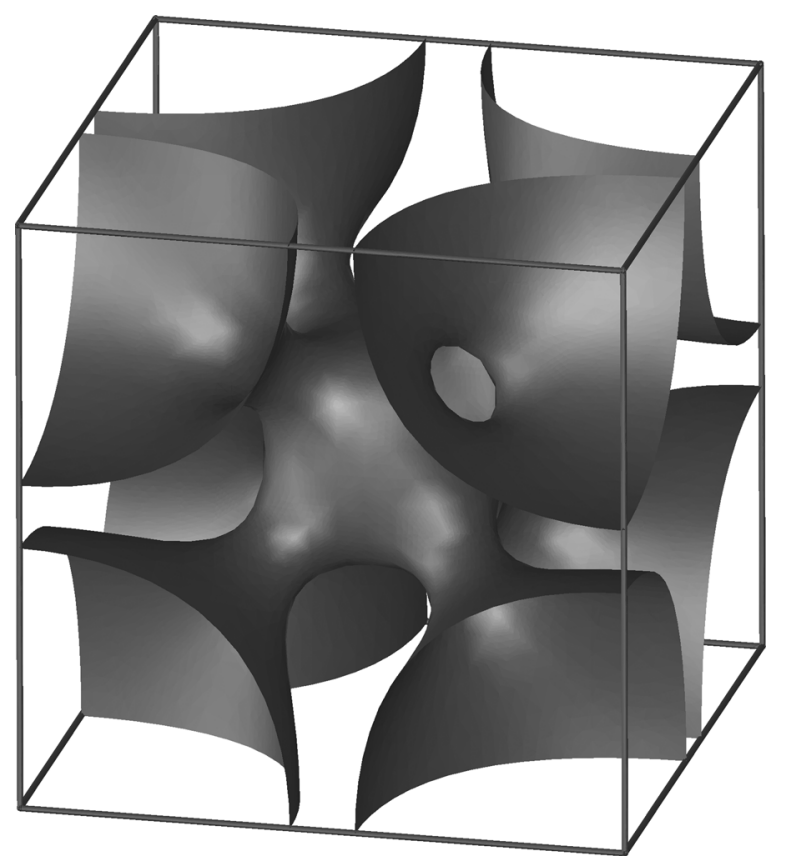

Figure 4: A periodic branched conformal cmc surface, visualized by K. Grosse-Brauckmann, University of Darmstadt, Germany.

$\int H^{2} \geq 8 \pi$. On the other hand, maximizers and minimizers of $\mathcal{F}_{q_{D}}$ satisfy $\int H^{2}=\lambda_{\min }^{+}{ }^{2} \leq 4 \pi$, hence these tori do not correspond to maximizers or minimizers.

Similar propositions also hold for immersions into $S^{3}$ and into hyperbolic space $H^{3}$, see [4]. We will only specify the case of $S^{3}=\mathrm{SU}(2)$.

Proposition 10.2. Assume that the Riemann spin surface $(M, g, \sigma)$ satisfies

$$
\lambda_{\min }^{+}(M,[g], \sigma)<2 \pi
$$

Let $a \in\left(0, \lambda_{\min }^{+}(M,[g], \sigma)\right.$ be given. Then there is a left periodic branched conformal cmc immersion $F: \widetilde{M} \rightarrow \mathrm{SU}(2)$ based on $(M, g)$. The mean curvature is equal to $H=\sqrt{\left(\lambda_{\min }^{+}(M,[g], \sigma) / a\right)^{2}-1}$, and the area of a fundamental domain is $a^{2}$. The regular homotopy class of $F$ is determined by the spin-structure $\sigma$. The indices of all branching points are even, and the sum 


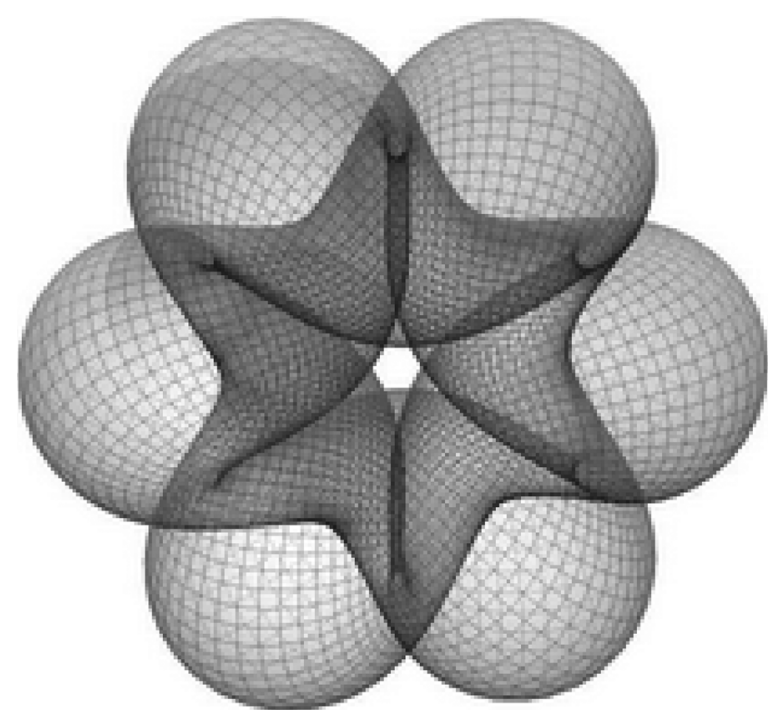

Figure 5: A twisty torus, a cmc immersed torus in $\mathbb{R}^{3}$, visualized by N. Schmitt, University of Tübingen, Germany.

of these indices is smaller than $2 \operatorname{genus}(M)$. In particular, if $M$ is a torus, there are no branching points.

An example where the image of the periodicity map has a finite image in $\mathrm{SU}(2)$ is given in figure 6 .

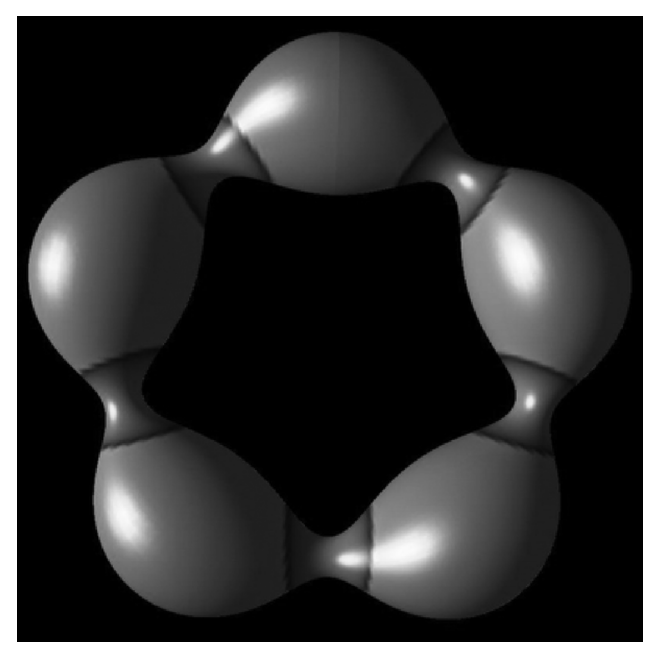

Figure 6: An unduloid in $S^{3}$, visualized by N. Schmitt, University of Tübingen, Germany. 
Remark. If $(M, g)$ is an analytic Riemannian manifold of dimension 3, then there is an analogue of the spinorial Weierstrass representation. This implies that solutions $\varphi$ of (2.3) can be geometrically interpreted as a conformal cmc embedding of $M \backslash \varphi^{-1}(0)$ into a (non-complete) four-dimensional Riemannian manifold $(N, h)$. This manifold $(N, h)$ carries a parallel spinor whose restriction to $(M, g)$ is again the solution of $(2.3)$. The manifold $(N, h)$ depends on $\varphi$ and is unique up to restriction to subsets and coverings. A more detailed exposition of this three-dimensional version is work in progress by the author.

\section{Appendix A. Elliptic regularity}

In this section we want to collect some facts about elliptic regularity for Dirac operators. The proofs of these statements are analogous to proofs of the corresponding statements for the Laplace operators as done, e.g., in $[1,17]$. Details on how to prove most statements of this section are given also in [4]. Either a proof is provided there or it is sketched how the statements are reduced to standard theorems.

Let $(N, g)$ be a Riemannian manifold, possibly with boundary, with a spin structure $\sigma$. The interior of $N$ is denoted as $N_{0}$. The spinor bundle $\Sigma(N, g, \sigma)$ is a complex vector bundle carrying a natural connection and a natural hermitian metric.

We now define the Sobolev norms of spinor fields.

Definition (Sobolev spaces). For any spinor $\psi$ smooth on $N$, and $q \in$ $(1, \infty), k \in \mathbb{N} \cup\{0\}$, we define the $H_{k}^{q}$-norm of $\psi$ as

$$
\|\psi\|_{H_{k}^{q}(N, g, \sigma)}=\sum_{l=0}^{k}\left(\int_{N}|\underbrace{\nabla}_{l-\text { times }^{\nabla \ldots \nabla}} \psi|^{q} \mathrm{dvol}_{g}\right)^{1 / q} .
$$

When the domain of integration is clear, we simply write $\|\psi\|_{H_{k}^{q}}$ instead of $\|\psi\|_{H_{k}^{q}(N, g, \sigma)}$. The closure of the space of smooth spinors with respect to this norms is denoted as $H_{k}^{q}(\Sigma(N, g, \sigma))$. If the underlying structure of Riemannian spin manifold is clear from the context, we often write shortly $H_{k}^{q}$, or in other situations where we want to emphasize the metric we write $H_{k}^{q}(g)$. We will also write $L^{q}$ for $H_{0}^{q}$.

Theorem A.1 (Interior $L^{p}$ estimates). Let $(N, g, \sigma)$ be a compact Riemannian spin manifold, possibly with boundary. Let $K$ be a compact subset 
of $N_{0}$. Let $\psi$ be in $H_{k}^{q}(\Sigma(N, g, \sigma))$ and let $\varphi \in H_{1}^{1}(\Sigma(N, g, \sigma))$ be a weak solution (i.e., in the sense of distributions) of

$$
D \varphi=\psi
$$

on $N_{0}$. Then $\left.\varphi\right|_{K} \in H_{k+1}^{q}(\Sigma(K, g, \sigma))$ and

$$
\|\varphi\|_{H_{k+1}^{q}(\Sigma(K, g, \sigma))} \leq C \cdot\left(\|\psi\|_{H_{k}^{q}(\Sigma(N, g, \sigma))}+\|\varphi\|_{L^{q}(\Sigma(N, g, \sigma))}\right)
$$

where $C=C(N, g, \sigma, K, k, q)$, i.e., $C$ depends only on $N, g, \sigma, K, k$ and $q$.

Furthermore, if a sequence of metrics $\left(g_{i}\right)_{i \in \mathbb{N}}$ converges in the $C^{\infty}$ topology to a Riemannian metric $g$, then the constants $C$ can be chosen such that

$$
\sup _{i \in \mathbb{N}} C\left(N, g_{i}, \sigma, K, k\right)<\infty
$$

In order to prove the theorem, it is sufficient to prove it in the case that $N$ and $K$ are small concentric geodesic balls, the radius being controlled in terms of curvature bounds, and bounds on derivatives of the curvature. As explained in [4] this can be done in a way analogous to the corresponding statement for the Laplacian in [17]. The general statement then can be reduced to the special case by covering (the general) $K$ by finitely many small open balls and an associated partition of unity.

In the special case that the boundary of $N$ is the empty set, and that $K=N=N_{0}$, then there is a stronger version, that will be used as well.

Theorem A.2 (Global $L^{p}$ estimates). Let $(N, g, \sigma)$ be a compact Riemannian spin manifold without boundary and $\psi \in H_{k}^{q}(\Sigma(N, g, \sigma))$. Then any weak solution $\varphi \in H_{1}^{1}(\Sigma(N, g, \sigma))$ of

$$
D \varphi=\psi
$$

satisfies $\varphi \in H_{k+1}^{q}(\Sigma(N, g, \sigma))$, and there is a constant $C=C(N, g, \sigma)$ such that

$$
\|\varphi\|_{H_{k+1}^{q}} \leq C\left(\|\psi\|_{H_{k}^{q}}+\left\|\pi_{\operatorname{ker}(D)}(\varphi)\right\|_{L^{q}}\right),
$$

where $\pi_{\operatorname{ker}(D)}$ is the $L^{2}$-orthogonal projection to the kernel of $D$.

The proof of this theorem is not difficult if one uses some facts about pseudodifferential operators, explained for example in [37]. We abbreviate $\pi:=\pi_{\operatorname{ker}(D)}$. The spectrum of the elliptic operator $D+\pi$ is bounded away from 0 , hence it is invertible and its inverse is a pseudodifferential operator 
of degree -1 . Pseudodifferential operators of degree -1 are continuous from $L^{q}$ to $H_{1}^{q}$. Hence,

$$
\begin{aligned}
\|\varphi\|_{H_{1}^{q}} & =\left\|(D+\pi)^{-1}(D+\pi) \varphi\right\|_{H_{1}^{q}} \leq C\|(D+\pi) \varphi\|_{L^{q}} \\
& \leq C\left(\|D \varphi\|_{L^{q}}+\|\pi(\varphi)\|_{L^{q}}\right)
\end{aligned}
$$

This is the statement of the theorem in the case $k=0$, and the statement for $k>0$ follows for example by using Theorem A.1.

However, a uniformity statement for converging $g_{i} \rightarrow g$ as in Theorem A.1 does not hold. If $\operatorname{dim} \operatorname{ker} D_{g}>\lim \sup _{i} \operatorname{dim} \operatorname{ker} D_{g_{i}}$, then one easily shows that there are eigenspinors $\varphi_{i}$ for $D_{g_{i}}$ to eigenvalues $\lambda_{i} \rightarrow 0, \lambda_{i} \neq 0$ with

$$
\frac{\left\|D \varphi_{i}\right\|_{L^{q}\left(g_{i}\right)}+\left\|\pi_{g_{i}}\left(\varphi_{i}\right)\right\|_{L^{q}\left(g_{i}\right)}}{\left\|\varphi_{i}\right\|_{H_{1}^{q}\left(g_{i}\right)}}=\lambda_{i}
$$

which would contradict a uniform version of Theorem A.2.

We also need some facts about Hölder norms.

Definition (Hölder spaces). Let $\alpha \in(0,1]$. On $C^{\infty}(\Sigma(N, g, \sigma))$ we define the Hölder norms

$$
\begin{aligned}
\|\varphi\|_{C^{0, \alpha}}:= & \|\varphi\|_{C^{0}}+\operatorname{höl}_{\alpha}(\varphi) \\
\|\varphi\|_{C^{1, \alpha}}:= & \|\varphi\|_{C^{0}}+\|\nabla \varphi\|_{C^{0, \alpha}}=\|\varphi\|_{C^{0}}+\|\nabla \varphi\|_{C^{0}}+\operatorname{höl}_{\alpha}(\nabla \varphi), \\
\operatorname{hol}_{\alpha}(Q):= & \sup \left\{\frac{\left|Q(x)-P_{\gamma} Q(y)\right|}{d(x, y)^{\alpha}} \mid x, y \in M, x \neq y, P_{\gamma}\right. \text { is the } \\
& \quad \text { parallel transport along a shortest geodesic } \gamma \text { from } x \text { to } y,\} .
\end{aligned}
$$

If the shortest geodesic is not unique, then we use the convention that the supremum runs over all possible choices of shortest geodesics.

The completions of $C^{\infty}(\Sigma(N, g, \sigma))$ with respect to the Hölder norms $C^{0, \alpha}$ and $C^{1, \alpha}$ define the Hölder spaces $C^{0, \alpha}=C^{0, \alpha}(\Sigma(N, g, \sigma))$ and $C^{1, \alpha}=$ $C^{1, \alpha}(\Sigma(N, g, \sigma))$.

Hölder norms are important as they admit Schauder estimates.

Theorem A.3 (Schauder estimates). Let $(N, g, \sigma)$ be a compact Riemannian spin manifold, possibly with boundary, and let $K$ be a compact subset of $N_{0}$. Suppose $\psi \in C^{k, \alpha}(\Sigma(N, g, \sigma)), k \in \mathbb{N} \cup\{0\}$. Then for any weak 
solution $\varphi \in L^{1}(\Sigma(N, g, \sigma))$ of

$$
D \varphi=\psi,
$$

we have $\left.\varphi\right|_{K} \in C^{k+1, \alpha}(\Sigma(K, g, \sigma))$ and

$$
\|\varphi\|_{C^{k+1, \alpha}(\Sigma(K, g, \sigma))} \leq C \cdot\left(\|\psi\|_{C^{k, \alpha}(\Sigma(N, g, \sigma))}+\|\varphi\|_{C^{0}(\Sigma(N, g, \sigma)}\right),
$$

where $C=C(N, g, \sigma, K, \alpha)$.

Furthermore, if a sequence of metrics $\left(g_{i}\right)_{i \in \mathbb{N}}$ converges in the $C^{\infty}$. topology to a Riemannian metric $g$, then the constants $C$ can be chosen such that

$$
\sup _{i \in \mathbb{N}} C\left(N, g_{i}, \sigma, K, k\right)<\infty .
$$

As before, one important special case is that $N$ has empty boundary and $K=N=N_{0}$.

The proof can be done in a way analogous to the proof of the corresponding statements for the Laplacian in [17]. Again it is sufficient to prove it for small concentric geodesic balls, and to glue them together. We omit the details. For a proof for concentric geodesic balls is provided for example in [4].

In local charts one easily reduces the following theorem to the standard Arcela-Ascoli theorem.

Theorem A.4 (Arcela-Ascoli). Let $(N, g, \sigma)$ be a Riemannian spin manifold, possibly with boundary. For $m \in \mathbb{N} \cup\{0\}$, the inclusion $C^{m, \alpha}(\Sigma(N, g, \sigma)) \rightarrow C^{m}(\Sigma(N, g, \sigma))$ is compact, i.e., a bounded sequence in $C^{m, \alpha}(\Sigma(N, g, \sigma))$ has a subsequence convergent in $C^{m}(\Sigma(N, g, \sigma))$.

Sobolev and Hölder spaces are related by several embedding theorems, some of them are compact, others are only bounded. We summarize the embeddings that are needed in the article.

Theorem A.5 (Embedding theorems). Let $k, s \in \mathbb{N} \cup\{0\}, k \geq s$ and $q, r \in(1, \infty)$. Let $(M, g, \sigma)$ be a compact Riemannian spin manifold without boundary. All spaces of functions are defined on sections of $\Sigma(M, g, \sigma)$.

(a) (Sobolev embedding theorem I). If

$$
\frac{1}{r}-\frac{s}{n} \geq \frac{1}{q}-\frac{k}{n}
$$

then $H_{k}^{q}$ is continuously embedded into $H_{s}^{r}$. 
(b) (Rellich-Kondrakov theorem). If strict inequality holds in (A.1) and if $k>s$, then the inclusion $H_{k}^{q} \hookrightarrow H_{s}^{r}$ is a compact map.

(c) (Sobolev embedding theorem II). Suppose $0<\alpha<1, m \in\{0,1\}$ and

$$
\frac{1}{q} \leq \frac{k-m-\alpha}{n}
$$

Then $H_{k}^{q}$ is continuously embedded into $C^{m, \alpha}$.

\section{Appendix B. Some facts about Hölder spaces}

In this section we want to include some proofs of probably well-known statements about Hölder spaces.

Lemma B.1. Let $V$ be a Euclidean vector space, $\alpha \in(0,1), m \in \mathbb{N}$. Then the map $V \rightarrow \bigotimes_{m} V$,

$$
T: x \mapsto|x|^{\alpha-m} \underbrace{x \otimes \cdots \otimes x}_{m \text { times }}
$$

is $C^{\alpha}$.

Proof. Let $x, y \in V$. At first, we suppose that $\|x-y\|<\delta$ and $\|x\| \geq \delta$. Then we calculate

$$
\|x\|\left\|\frac{x}{\|x\|}-\frac{y}{\|y\|}\right\|=\left\|x-\frac{\|x\|}{\|y\|} y\right\| \leq\|x-y\|+\left|1-\frac{\|x\|}{\|y\|}\right|\|y\| \leq 2 \delta
$$

Hence

$$
\left\|\frac{x}{\|x\|} \otimes \cdots \otimes \frac{x}{\|x\|}-\frac{y}{\|y\|} \otimes \cdots \otimes \frac{y}{\|y\|}\right\| \leq \frac{2 \delta m}{\|x\|}
$$

This implies

$$
\begin{aligned}
|T(x)-T(y)| & \leq\left|\|x\|^{\alpha}-\|y\|^{\alpha}\right|+\|x\|^{\alpha} \frac{2 \delta m}{\|x\|} \\
& \leq\|x-y\|^{\alpha}+\|x\|^{\alpha-1} 2 \delta m \\
& \leq(2 m+1) \delta^{\alpha} .
\end{aligned}
$$

Now suppose that $\|x-y\|<\delta$ and $\|x\|<\delta$. Then

$$
|T(x)-T(y)| \leq\|x\|^{\alpha}+\|y\|^{\alpha} \leq 3 \delta^{\alpha} .
$$


Hence, we obtain $|T(x)-T(y)| \leq(2 m+1)\|x-y\|$ for all $x, y \in V$, and hence $T$ is $C^{\alpha}$.

Proposition B.1. If $V$ is a vector bundle over a compact Riemannian manifold, and if $\varphi$ is a $C^{1, \alpha}$-section of $V, \alpha \in(0,1)$ then $\psi=|\varphi|^{\beta} \varphi, \beta>0$ is a $C^{1, \gamma}$-section for $\gamma:=\min \{\alpha, \beta\}$.

Proof. The section $\varphi$ is obviously Lipschitz, hence $|\varphi|^{\beta}$ is $C^{\beta}$. We have to show that

$$
\nabla \psi=|\varphi|^{\beta} \nabla \varphi+\beta\langle\nabla \varphi, \varphi\rangle|\varphi|^{\beta-2} \varphi
$$

is $C^{\gamma}$. The first summand is a product of $C^{\beta}$ and $C^{\alpha}$, hence $C^{\gamma}$. According to the previous lemma, $|\varphi|^{\beta-2} \varphi \otimes \varphi$ is $C^{\beta}$, hence the second summand is a product of $C^{\alpha}$ and $C^{\beta}$, hence also $C^{\gamma}$.

\section{Acknowledgments}

I am very much indebted to Christian Bär for various support. Thank you also to Emmanuel Humbert for his continuing interest in conformal spin geometry and his deep analytical ideas. Many electronic and personal discussions with Robert Kusner and Karsten Grosse-Brauckmann were also very helpful. I also want to thank B. Booss-Bavnbek, Oussama Hijazi, Sebastian Montiel, Sergiu Moroianu, Victor Nistor, Reiner Schätzle and Guofang Wang for several stimulating discussions related to this article. Thanks also to Simon Raulot and Nadine Große for their interest in the preprint version of this article.

\section{References}

[1] R. Adams, Sobolev spaces, Pure and Applied Mathematics, 65, Academic Press, New York-London, 1975.

[2] B. Ammann, Spin-Strukturen und das Spektrum des Dirac-operators, $\mathrm{PhD}$ thesis, University of Freiburg, Germany, 1998, Shaker-Verlag Aachen 1998, ISBN 3-8265-4282-7.

[3] B. Ammann, A spin-conformal lower bound of the first positive Dirac eigenvalue, Diff. Geom. Appl. 18 (2003), 21-32.

[4] B. Ammann, A variational problem in conformal spin geometry, Habilitationsschrift, Universität Hamburg, 2003. 
[5] B. Ammann and E. Humbert, The spinorial $\tau$-invariant and 0-dimensional surgery, J. Reine Angew. Math. 624 (2008), 27-50.

[6] B. Ammann, E. Humbert and B. Morel, Mass endomorphism and spinorial Yamabe type problems, Comm. Anal. Geom. 14 (2006), 163-182.

[7] B. Ammann, J.-F. Grosjean, E. Humbert and B. Morel, A spinorial analogue of Aubin's inequality, Math. Z. 260 (2008), 127-151.

[8] T. Aubin, Équations différentielles non linéaires et problème de Yamabe concernant la courbure scalaire., J. Math. Pur. Appl. IX. Ser. 55 (1976), 269-296.

[9] C. Bär, Lower eigenvalue estimates for Dirac operators, Math. Ann. 293 (1992), 39-46.

[10] C. Bär, On nodal sets for Dirac and Laplace operators, Comm. Math. Phys. 188 (1997), 709-721.

[11] C. Bär, Extrinsic bounds for eigenvalues of the Dirac operator, Ann. Global Anal. Geom. 16 (1998), 573-596.

[12] A. L. Besse, Einstein manifolds, Ergebnisse der Mathematik und ihrer Grenzgebiete, 3. Folge, 10, Springer-Verlag, Berlin, 1987.

[13] J.-P. Bourguignon and P. Gauduchon, Spineurs, opérateurs de Dirac et variations de métriques, Comm. Math. Phys. 144 (1992), 581-599.

[14] T. Friedrich, Der erste Eigenwert des Dirac-Operators einer kompakten Riemannschen Mannigfaltigkeit nicht-negativer Krümmung, Math. Nach. 97 (1980), 117-146.

[15] T. Friedrich, On the spinor representation of surfaces in Euclidean 3-space, J. Geom. Phys. 28 (1998), 143-157.

[16] T. Friedrich, Dirac operators in Riemannian geometry, Graduate Studies in Mathematics, 25, AMS, Providence, 2000.

[17] D. Gilbarg and N. Trudinger, Elliptic partial differential equations of second order, Grundlehren der mathematischen Wissenschaften, 224, Springer-Verlag, Berlin, New York, 1977.

[18] O. Hijazi, A conformal lower bound for the smallest eigenvalue of the Dirac operator and Killing spinors, Comm. Math. Phys. 104 (1986), 151-162. 
[19] O. Hijazi, Spectral properties of the Dirac operator and geometrical structures, in 'Geometric Methods for Quantum Field Theory. Proceedings of the Summer School', eds. H. Ocampo et al., Villa de Leyva, Colombia, July 12-30, 1999, World Scientific, Singapore, 2001, 116-169.

[20] N. Hitchin, Harmonic spinors, Adv. Math. 14 (1974), 1-55.

[21] K.-D. Kirchberg, An estimation for the first eigenvalue of the Dirac operator on closed Kähler manifolds of positive scalar curvature, Ann. Global Anal. Geom. 4 (1986), 291-325.

[22] K.-D. Kirchberg, Compact six-dimensional Kähler spin manifolds of positive scalar curvature with the smallest possible first eigenvalue of the Dirac operator, Math. Ann. 282 (1988), 157-176.

[23] R. Kusner and N. Schmitt, The spinor representation of surfaces in space, Preprint, http://www.arxiv.org/abs/dg-ga/9610005, 1996.

[24] W. Kramer, U. Semmelmann and G. Weingart, The first eigenvalue of the Dirac operator on quaternionic Kähler manifolds, Comm. Math. Phys. 199 (1998), no. 2, 327-349.

[25] W. Kramer, U. Semmelmann and G. Weingart, Eigenvalue estimates for the Dirac operator on quaternionic Kähler manifolds, Math. Z. 230 (1999), 727-751.

[26] A. Lichnerowicz, Spineurs harmoniques, C. R. Acad. Sci. Paris 257 (1963), 7-9.

[27] J. Lott, Eigenvalue bounds for the Dirac operator, Pacific J. Math. 125 (1986), 117-126.

[28] H.-B. Lawson and M.-L. Michelsohn, Spin geometry, Princeton University Press, Princeton, 1989.

[29] J.M. Lee and T.H. Parker, The Yamabe problem, Bull. Am. Math. Soc. New Ser. 17 (1987), 37-91.

[30] P. Li and S.-T. Yau, A new conformal invariant and its applications to the Willmore Conjecture and the first eigenvalue of compact surfaces, Invent. Math. 69 (1982), 269-291.

[31] S. Maier, Generic metrics and connections on spin- and spin- ${ }^{{ }_{-}}$ manifolds, Comm. Math. Phys. 188 (1997), 407-437. 
[32] B. Morel, Surfaces in $S^{3}$ and $H^{3}$ via spinors, Actes de Séminaire de Théorie Spectrale et Géométrie, Univ. Grenoble I, Année 2004-2005, 23 (2005), 131-144

[33] F. Pfäffle, Eigenwertkonvergenz für Dirac-Operatoren, PhD thesis, University of Hamburg, Germany, 2002, Shaker Verlag, Aachen, 2003, ISBN 3-8322-1294-9.

[34] U. Pinkall, Regular homotopy classes of immersed surfaces, Topology 24 (1985), 421-434.

[35] J. Roe, Elliptic operators, topology and asymptotic methods, Pitman Research Notes in Mathematics Series, 179, Pitman, Longman, 1988.

[36] N. Schmitt, Minimal surface with planar embedded ends, PhD dissertation, University of Amherst, 1993.

[37] M. E. Taylor, Pseudodifferential operators, Princeton University Press, Princeton, NJ, 1981.

[38] L. Voss, Eigenwerte des Dirac-Operators auf Hyperflächen, Diplomarbeit, Humboldt Universität zu Berlin, 1999.

[39] K. Weierstrass, Untersuchungen über die Flächen, deren mittlere Krümmung überall gleich Null ist, available in Weierstrass, Mathematische Werke III, 1866, 39-52.

NWF I - MATHEMATIK

UNIVERSitÄT REgENSBURG

93040 REgENSBURG

GERMANY

E-mail address: bernd.ammann@gmx.de

WWW: http://www. berndammann.de

ReCEIVEd May 12, 2003 
